ARTICLE

\title{
OPTN is a host intrinsic restriction factor against neuroinvasive HSV-1 infection
}

\author{
Joshua Ames 1,2, Tejabhiram Yadavalli (i) 2, Rahul Suryawanshi², James Hopkins ${ }^{1,2}$, Alexander Agelidis (10 1,2,
}

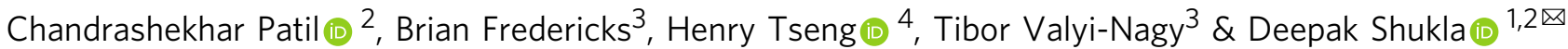

Fast-replicating neurotropic herpesviruses exemplified by herpes simplex virus-1 (HSV-1) naturally infect the central nervous system (CNS). However, most individuals intrinsically suppress the virus during a primary infection and preclude it from significantly damaging the CNS. Optineurin (OPTN) is a conserved autophagy receptor with little understanding of its role in neurotropic viral infections. We show that OPTN selectively targets HSV-1 tegument protein, VP16, and the fusion glycoprotein, gB, to degradation by autophagy. OPTN-deficient mice challenged with HSV-1 show significant cognitive decline and susceptibility to lethal CNS infection. OPTN deficiency unveils severe consequences for recruitment of adaptive immunity and suppression of neuronal necroptosis. Ocular HSV-1 infection is lethal without OPTN and is rescued using a necroptosis inhibitor. These results place OPTN at the crux of neuronal survival from potentially lethal CNS viral infections.

\footnotetext{
${ }^{1}$ Department of Microbiology and Immunology, University of Illinois at Chicago, College of Medicine, Chicago, IL, USA. ${ }^{2}$ Department of Ophthalmology and Visual Sciences, University of Illinois at Chicago, College of Medicine, Chicago, IL, USA. ${ }^{3}$ Department of Pathology, University of Illinois at Chicago, College of Medicine, Chicago, IL, USA. ${ }^{4}$ Duke Eye Center and Department of Ophthalmology, Duke University Medical Center, Durham, NC, USA.

凶email: dshukla@uic.edu
} 
erpesviruses cause widespread infections that can lead to encephalitis, and evidence suggests herpesvirus infections of the central nervous system (CNS) contribute to the complex etiology of neurodegenerative disorders of the brain and eye $\mathrm{e}^{1-5}$. Immunocompetent individuals are protected, but defects in innate or adaptive immunity may lead to death of infected neurons ${ }^{6,7}$. We highlight Optineurin (OPTN)-dependent selective autophagy as a critical intrinsic immune barrier against neurotropic viruses. Selective autophagy is required for specific degradation of damaging cellular occupants and is implicated in protecting the CNS from neurodegenerative diseases ${ }^{8-10}$.

Intracellular cargoes are ubiquitinated for binding to autophagy receptors, and the affinity of the receptors are enhanced through phosphorylation by TANK binding kinase 1 (TBK1) ${ }^{11-13}$. OPTN is an autophagy receptor which facilitates degradation of mitochondria and bacteria ${ }^{11,14,15}$. Mutations in OPTN have been identified, implicating it in human diseases including glaucoma, amyotrophic lateral sclerosis (ALS), Huntington's disease, and Alzheimer's disease (AD) $)^{5,16-18}$. Evidence supports the protective role of autophagy against protein aggregation in neurons, and it is suggested that it is an innate immune defense mechanism of the CNS ${ }^{19,20}$.

Selective autophagy can regulate signaling pathways ${ }^{8,21-23}$. OPTN negatively regulates necroptosis by degrading receptor interacting serine/threonine kinase 1 (RIPK1), protecting against neurodegeneration ${ }^{24}$. Autophagy has been associated with protection from interferon gamma (IFN $\gamma$ ) and tumor necrosis factoralpha (TNF- $\alpha$ ) mediated cell death ${ }^{25}$. This potential for selective autophagy to regulate inflammation and cell death may influence the outcome of CNS infections. Additionally, herpesviruses express factors to circumvent autophagy. HSV-1 encodes a virulence factor, $\gamma_{1} 34.5$ to inhibit autophagy ${ }^{26}$. Our study uses Optn-/ - in vitro and in vivo models to examine the role of OPTN in the context of CNS immunity.

In this work we show that the host protein, OPTN, restricts HSV-1 infection in vitro and in vivo. OPTN deficiency leads to increased levels of the essential HSV-1 proteins, VP16, and gB. Furthermore, OPTN deficiency leads to diminished host immune responses to ocular HSV-1 infection, increased susceptibility to herpes encephalitis, and long-term loss of cognition.

\section{Results}

OPTN restrict spread of HSV-1. Optn-/- HeLa cells support rapid spread and replication of HSV-1. Time-lapse microscopy of GFP expressing HSV-1 revealed spread of infection to a larger population of cells in Optn-/- relative to Optn+/+ cells (Fig. 1a, b). By $24 \mathrm{hpi}$ the production of infectious virus and viral genome replication was increased Optn-/- cells by $\sim 100$-fold and 5 -fold respectively because of the enhanced viral spread (Fig. 1c, f). The percentage of infected cells was higher in the Optn-I- cell line 24 hpi (Fig. 1d, e) but entry of virus into cells was similar for both Optn-l- and Optn+l+ (Fig. 1g), thus ruling out different entry rates as an explanation for the increased viral spread. To further support the role of OPTN, mouse embryonic fibroblast (MEF) cells were derived from Optn+/+ or $-/-$ mouse strains. Optn-/- MEF cells expressed significantly more late gene promoter signal when infected with HSV-1 KOS-strain (Supplementary Fig. 2A-C). When infected with HSV-1 17-strain, Optn-/- MEF cells showed significantly more widespread infection (Supplementary Fig. 2D-E). These results were further supported by infection of human corneal epithelial (HCE) cells and Lund human mesencephalic (LUHMES) cells that had been transfected with an siRNA targeting OPTN expression (Supplementary Fig. 1). Using multiple HSV-1 strains and pseudorabies virus (PRV), all expressing fluorescent reporters, we observed that OPTN generally restricts the spread of alphaherpesviruses in vitro. However, the differentiated neurons derived from LUHMES showed strain and virus specific differences in reporter signal. HCE, but not LUHMES, cells showed significantly more HSV-1 17 strain infection when OPTN was knocked down (Supplementary Fig. 2F-J). When infected with a dual reporter HSV1 KOS strain, the reporter for HSV-1 late gene expression was significantly more expressed in both HCE and LUHMES cells where OPTN was knocked down, but only the LUHMES cells showed significantly more early gene reporter expression (Supplementary Fig. 2K-P). When infected with PRV expressing GFP under a CMV promoter, and RFP fused to the capsid protein, only capsid levels were significantly higher in OPTN knockdown HCE cells (Supplementary Fig. 2Q-V). Overall OPTN restricts infection, and this trend was observed for multiple $\mathrm{HSV}-1$ strains and PRV in diverse cell types.

OPTN is required for selective degradation of essential HSV-1 proteins. OPTN has been established as an autophagy receptor for the selective autophagy of bacteria and organelles, therefore we hypothesized diminished viral protein levels due to selective autophagy against HSV-1. Optn $+/+$ or $-/-$ cells were infected with HSV-1 for $12 \mathrm{~h}$ prior to addition of cycloheximide (CHX) to inhibit protein synthesis. Optn-/- cells expressed significantly higher levels of VP16 and gB, but not ICP0 (Fig. 2a, b). The levels of ICP0, VP16, and gB decreased over time for both genotypes despite the much higher levels of viral protein. This trend was the same for gB expression in HCE cells (Fig. 2c, d), and both gB and VP16 were detected in the central nervous system of HSV-1 infected Optn-/- mice but not Optn+/+ mice (Fig. 2e). To determine if the degradation of the proteins that were suppressed in an OPTN-dependent manner was autophagy dependent Optn+/+ cells were infected with HSV-1 for $8 \mathrm{~h}$ prior to the addition of CHX and either Bafilomycin A1 (BafA1) to inhibit autophagy, MG132 to inhibit the proteosome, or DMSO as a vehicle control. After $24 \mathrm{~h}$ of infection the $8 \mathrm{~h}$ starting level, $24 \mathrm{~h}$ with BafA1, and $24 \mathrm{~h}$ with MG132 protein levels were compared to the $24 \mathrm{hr}$ DMSO levels. In Optn+/+ cells, ICP0 levels were significantly higher than DMSO treatment in the MG132 treatment group, VP16 levels were significantly higher than DMSO treatment in both BafA1 and MG132 treatment groups, and gB levels were significantly higher than DMSO treatment in the BafA1 treatment group (Fig. 3a, b). This trend was the same for HCE cells (Fig. 3c, d), and for the degradation of $g B$ in LUHMES cells (Fig. 3e, f). This shows that degradation of gB and VP16 is autophagy dependent, and the levels of gB and VP16 are suppressed in an OPTN-dependent manner. Furthermore, superresolution microscopy of cells infected with a GFP-VP16 fusion protein expressing HSV-1 strain revealed the association and staining overlap of OPTN stained aggregates with VP16 spherical structures which measured $\sim 250 \mathrm{~nm}$ in diameter (Fig. 4a). Immunofluorescent staining also showed the strongly colocalized signal between OPTN, VP16, and the lysosome marker, lysosomal-associated membrane protein 1 (LAMP1), by which OPTN is associated with VP16 outside of the nucleus (Fig. 4b, c). TBK1 phosphorylation of OPTN at residue serine-177 is reported to regulate OPTN autophagy receptor activity ${ }^{13}$. Coimmunoprecipitation of OPTN showed that upon infection OPTN first interacted with TBK1, and then with VP16 (Fig. 4d). When TBK1 was inhibited using BX795, both the total level of OPTN and the phosphorylated form were decreased in an infection independent manner (Fig. 4e) suggesting that OPTN function is constitutively regulated by TBK1. This function of OPTN does not affect the total level of autophagy in HeLa or LUHMES cells as is reflected by the lack of increased or decreased LC3 lipidation, the process by which LC3 is recruited to 

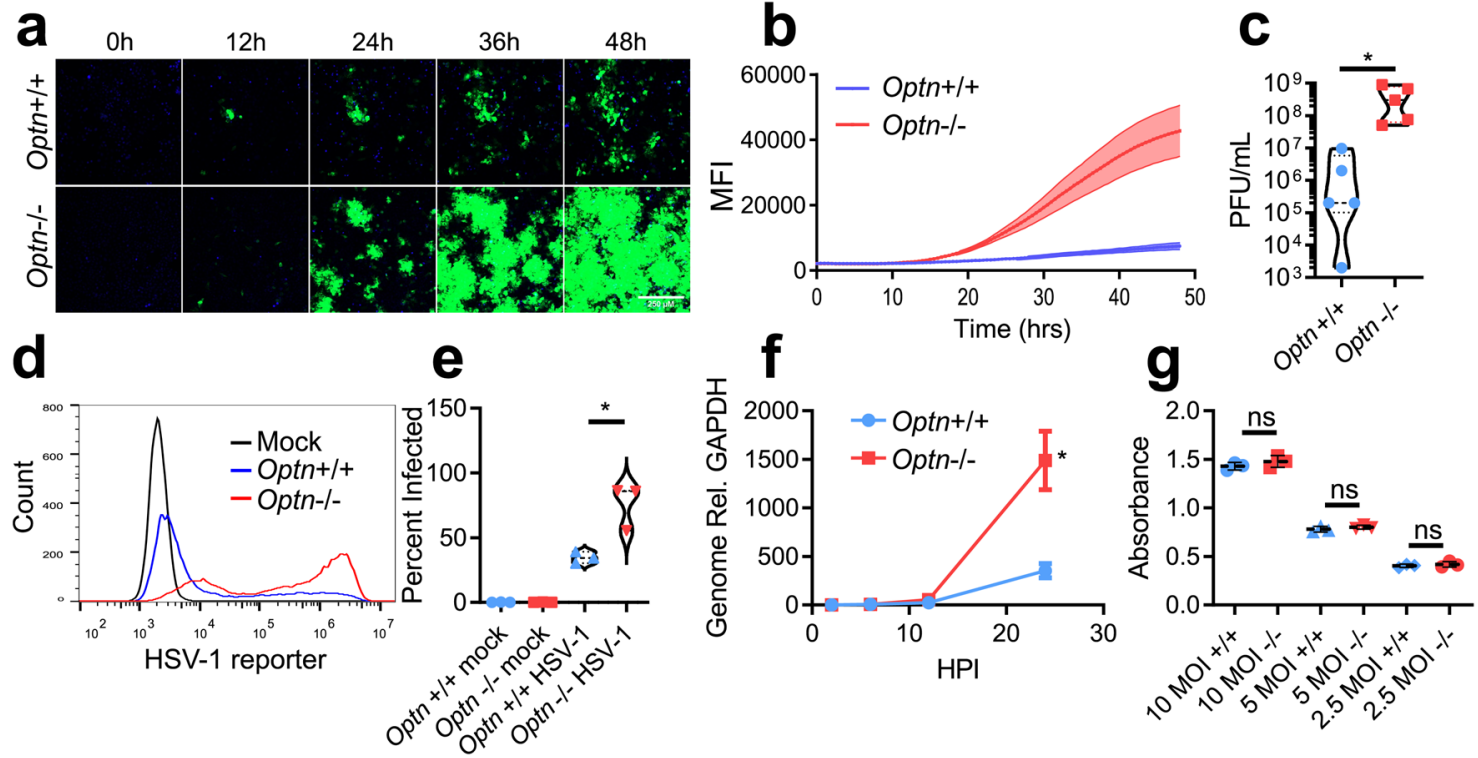

Fig. 1 OPTN restricts spread of HSV-1. a Representative time-lapse imaging of Optn+/+ and -/- cells infected with GFP-HSV-1 17-strain at 0.1 MOI for $48 \mathrm{~h}$. Time interval was $30 \mathrm{~min}$. Scale bar is $250 \mu \mathrm{m}$. b Quantification of average time-lapse fluorescent intensity over time for three replicates. c Violin plot of Optn $+/+$ and $-/-$ cells infected with HSV-1 at $0.1 \mathrm{MOI}$ and assayed for plaques 24 hpi. $n=5$ independent replicates per group. $P=0.0446$. d, e (left) Histogram of Optn $+/+$ and $-/$ - cells infected with GFP- HSV-1 at $0.1 \mathrm{MOI} 24$ hpi measured by flow cytometry, (right) violin plot of percentage of cells infected with GFP-HSV-1. $n=3$ independent replicates per group. $P=0.0174$. f Genomes present in Optn+/+ and $-/-$ cells infected with GFP- HSV-1 at $0.1 \mathrm{MOI} . n=3$ independent replicates per group. $P=0.0220$. $\mathbf{g}$ Colorimetric readout of replication deficient and $\beta$-galactosidase expressing HSV-1 infected Optn $+/+$ and $-/-$ cells at multiple MOls. $n=3$ independent replicates per group. $P=0.3083(10 \mathrm{MOI}), P=0.3575(5 \mathrm{MOI}), P=0.4945(2.5 \mathrm{MOI})$. Data are presented as mean values $\pm \operatorname{SEM}(\mathbf{b}, \mathbf{f})$. Two-tailed Student's $t$ test was performed for statistical analysis $(\alpha=0.05)$. $p<0.05 ;$ ns not significant. Source data are provided as a Source Data file.

autophagosomal membranes, during infection (Supplementary Fig. 3A-D). HSV-1 is known to inhibit host macroautophagy through the virulence factor, $\gamma_{1} 34.5$. While OPTN deficiency resulted in increased infection by wildtype HSV-1, infection with a $\gamma_{1} 34.5$ null mutant of HSV-1 resulted in equal levels of infection between both genotypes (Supplementary Fig. 3E). Together this data suggests that OPTN is a constitutively active antiviral defense factor that suppresses the expression of autophagy degradable essential viral proteins without changes to the total level of autophagy during infection.

OPTN is neuroprotective against herpes simplex virus encephalitis. To confirm the relevance of our in vitro results, we used an Optn-/mouse model for HSV-1 infection. Infection of Optn+/+ and Optn $-/-$ mice revealed striking differences in severity of infection. The infection spread to the trigeminal ganglion (TGN) of both mouse strains, but significant infection of the brainstem and brain only occurred in Optn-I- mice as observed by tissue viral titers (Fig. 5a-c). Optn-/- mice experienced significant decrease in weight and increased mortality compared to Optn $+/+$ mice (Fig. 5d, e). Neuron death was nearly undetectable in Optn+/+ brainstem tissue, but was high in Optn-/ - tissue, indicating that OPTN protects the CNS from HSV-1 neuro-invasion and neurodegeneration during acute infection (Fig. 5f, g). Human brainstem tissue from a normal brain, an ALS patient, and a herpes simplex virus encephalitis (HSE) patient were stained for OPTN (Fig. 5h). OPTN aggregates were absent in the control tissue but were present in the ALS tissue which is consistent with previously published reports ${ }^{17,18}$. In the HSE tissue we found widespread OPTN stained cytosolic aggregates, implicating a similar pathology in herpetic human diseases. Ultimately the damage to neurons in Optn-/- mice resulted in behavioral indication of neurodegeneration. In a novel object recognition test, Optn-1- animals showed a preference to explore a novel object without infection, but 30dpi Optn-/- animals did not exhibit the same preference, which is a known indicator for loss of cognition related to memory (Fig. $5 \mathrm{i}-\mathrm{k})^{27-29}$.

OPTN deficiency impairs host immunity. Immunity to HSV-1 depends partly on recruitment of CD8 T cells to the site of infection? Through cytokine profiling we revealed dramatic differences in the inflammatory state of Optn-/ - mice. The Optn-/- draining lymph nodes (dLNs) had less pro-inflammatory cytokine expression apart from IFN $\gamma$, and the overall cytokine profiles diverged between the two genotypes (Fig. 6a and Supplementary Fig. 4A). This is reflected in the lack of lymphadenopathy in the Optn-/- mice (Supplementary Fig. 4C, D). There was more expression of proinflammatory markers in Optn-/- mouse brainstems, suggesting greater neuroinflammation (Fig. $6 \mathrm{~b}$ and Supplementary Fig. 4B). This was accompanied by fewer $\mathrm{CD} 3+\mathrm{CD} 8+$ and $\mathrm{CD} 3+\mathrm{CD} 4+$ cells in brainstems of Optn-1- animals during infection (Fig. 6c-e). Despite the increased viral load in the Optn-/- mice, they had a stunted immune response through poor $\mathrm{dLN}$ activation and diminished $\mathrm{T}$ cell recruitment to the site of infection. Curiously, there was increased expression of IFN $\gamma$ in the Optn-/- mice in both tissues examined.

OPTN suppresses necroptosis in vitro and in neurons during HSV-1 infection. Necroptosis is suggested to be an antiviral response, but also a cause of neurodegeneration when it occurs in neuronal tissues ${ }^{24}$. OPTN negatively regulates necroptosis by targeting RIPK1 for autophagic degradation ${ }^{24}$. Pharmacological inhibition of autophagy or single gene knockout of essential autophagy genes, Atg5, Fip200, Atg16l1, or Becn1 are reported to protect cells from necroptosis mediated through IFN $\gamma$, TNF- $\alpha$, and RIPK $1^{25}$. Having shown that CNS infection leads to neuron death, and IFN $\gamma$ was highly represented in multiple tissues of Optn-/- mice, we sought to understand the role of OPTN in necroptosis during infection. Annexin $\mathrm{V}$ and propidium iodide staining revealed an OPTN-dependent divergence in cell death 

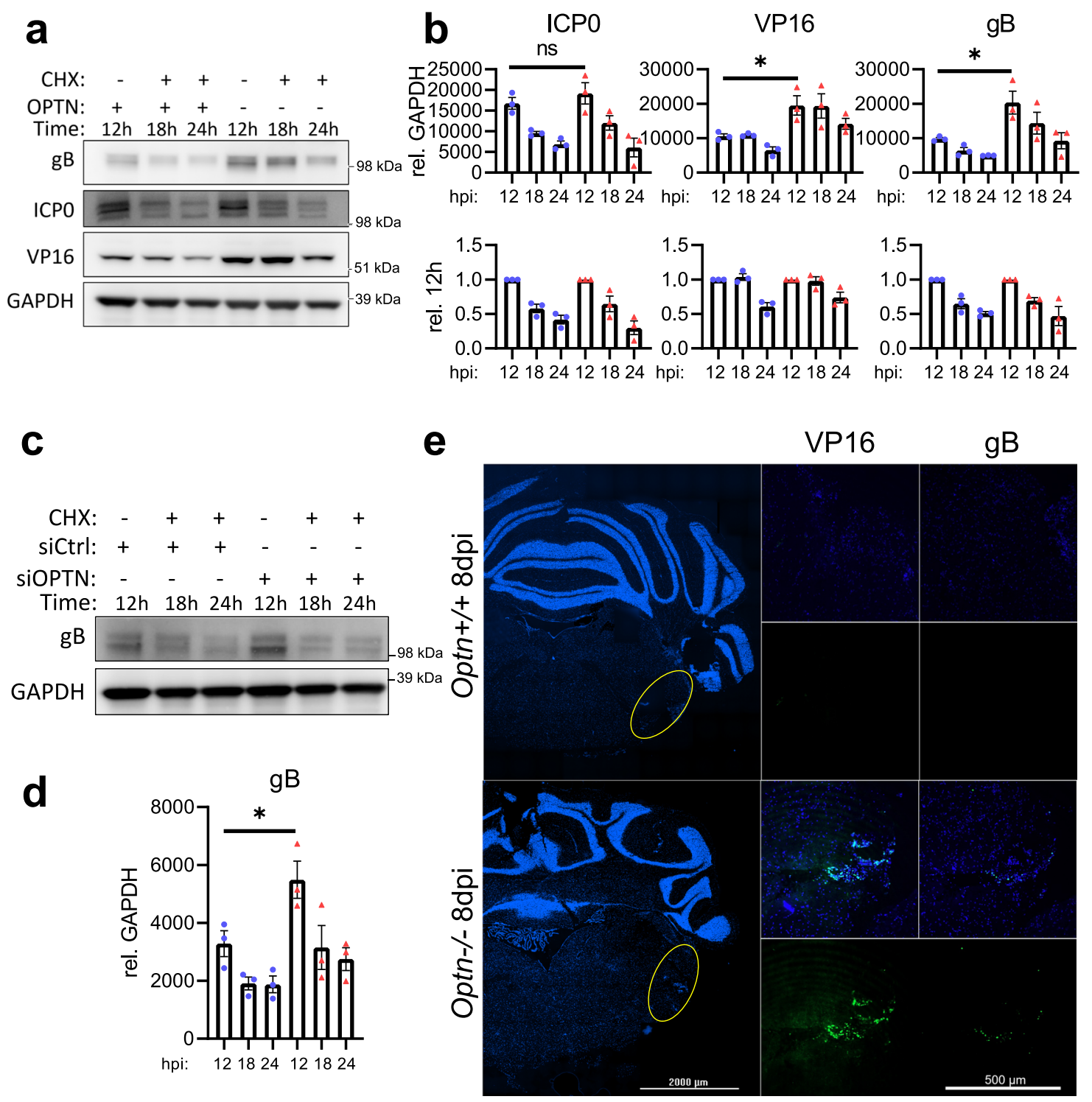

Fig. 2 OPTN suppresses expression of essential HSV-1 proteins VP16 and gB, but not ICPO. Optn $+/+$ and $-/-$ HeLa cells or siOPTN and siCtrl transfected HCE cells were infected at $1 \mathrm{MOI}$ with HSV-1 17-strain for $12 \mathrm{~h}$ before $\mathrm{CHX}$ addition to block protein synthesis, then cells were sampled at $12 \mathrm{~h}$, $18 \mathrm{~h}$, and $24 \mathrm{~h}$ after infection. a Shown for HeLa cells are immunoblots against the HSV-1 proteins gB, ICPO, VP16, and the internal reference GAPDH, b with band quantification relative to GAPDH or relative to initial normalized protein levels $(n=3$ replicates). $P=0.4591($ ICP0), $P=0.0368(V P 16), P=0.0347$ (gB). c Shown for HCE cells are immunoblots against the HSV-1 protein $\mathrm{gB}$ and the internal reference GAPDH, $\mathbf{d}$ with band quantification relative to GAPDH ( $n=3$ replicates). $P=0.0475$. e Representative staining for VP16 and gB in brainstems of HSV -1 infected Optn $+/+$ or $-/-$ mice 8 dpi. Data are presented as mean values \pm SEM $(\mathbf{b}, \mathbf{d})$. Two-tailed Student's $t$ test was performed for statistical analysis $(\alpha=0.05) . * p<0.05 ;$ ns not significant. Source data are provided as a Source Data file.

pathways, whereby OPTN removal shifted an apoptotic response to a necrotic response (Fig. 7a-c). RIPK1 inhibitor, Necrostatin$1 \mathrm{~s}$ (Nec-1s), rescued Optn-/- primary neurons from HSV-1induced death (Fig. $7 \mathrm{~d}$, e). Similar results were observed in vivo where Nec-1s treatment rescued Optn-/- mice survival, weight, viral load, and lymphadenopathy (Fig. $7 \mathrm{f}-\mathrm{i}$ ). These results confirmed that the increased cell death was RIPK1-dependent which is a hallmark of necroptosis ${ }^{24}$. Our findings suggest excess necroptosis may not be a beneficial innate defense mechanism during HSE and may be deleterious instead. It is interesting that inhibition of RIPK1 recovered the lymphadenopathy of Optn-/animals, and may indicate there is OPTN-dependent crosstalk between the IFN $\gamma$, TNF- $\alpha$, RIPK1 axis, and autophagy machinery during infection ${ }^{25}$.

\section{Discussion}

Previous reports outline mechanisms by which organelles and bacteria are degraded through OPTN-mediated selective autophagy ${ }^{13-15}$. We have shown that these mechanisms also apply to HSV-1 infection, revealing what is likely an evolutionarily conserved anti-herpesvirus intrinsic cellular defense. OPTN is regulated by TBK1 during infection, as inhibition of TBK1 inhibits the expression and phosphorylation of OPTN during HSV-1 infection. We also demonstrated the increased association of OPTN and TBK1 during infection, which was followed by association of OPTN and VP16. Additionally, we show that OPTN suppression of HSV-1 protein expression is selective for autophagy degradable viral factors, as gB and VP16 expression were significantly lower in wildtype cells, but ICP0 
a
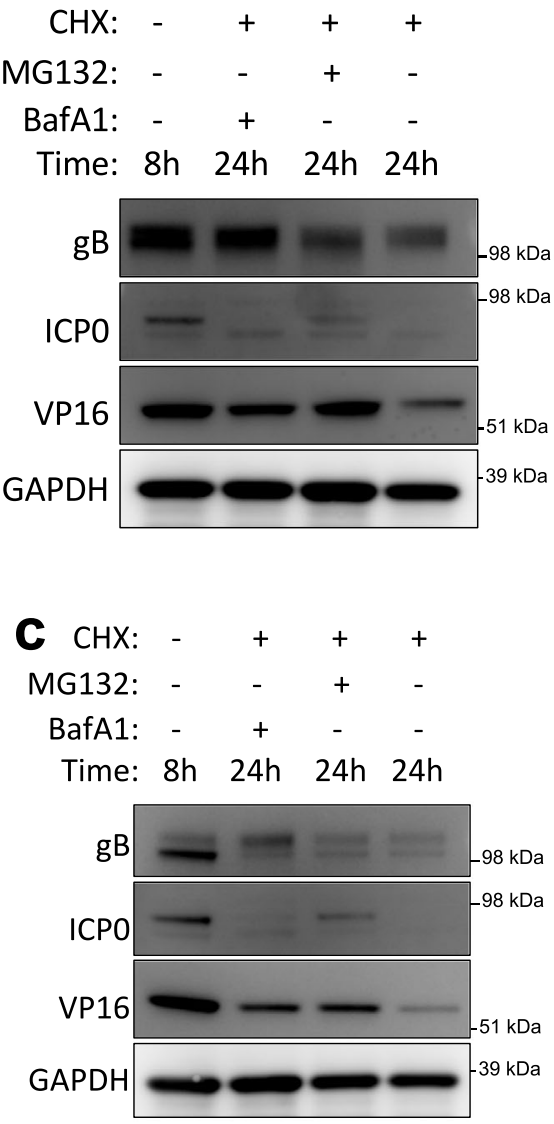

e

$$
\begin{array}{rcccc}
\text { CHX: } & - & + & + & + \\
\text { MG132: } & - & - & + & - \\
\text { BafA1: } & - & + & - & - \\
\text { Time: } & 8 \mathrm{~h} & 24 \mathrm{~h} & 24 \mathrm{~h} & 24 \mathrm{~h}
\end{array}
$$

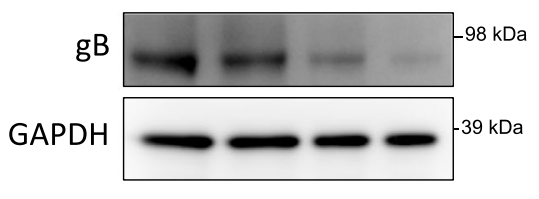

b
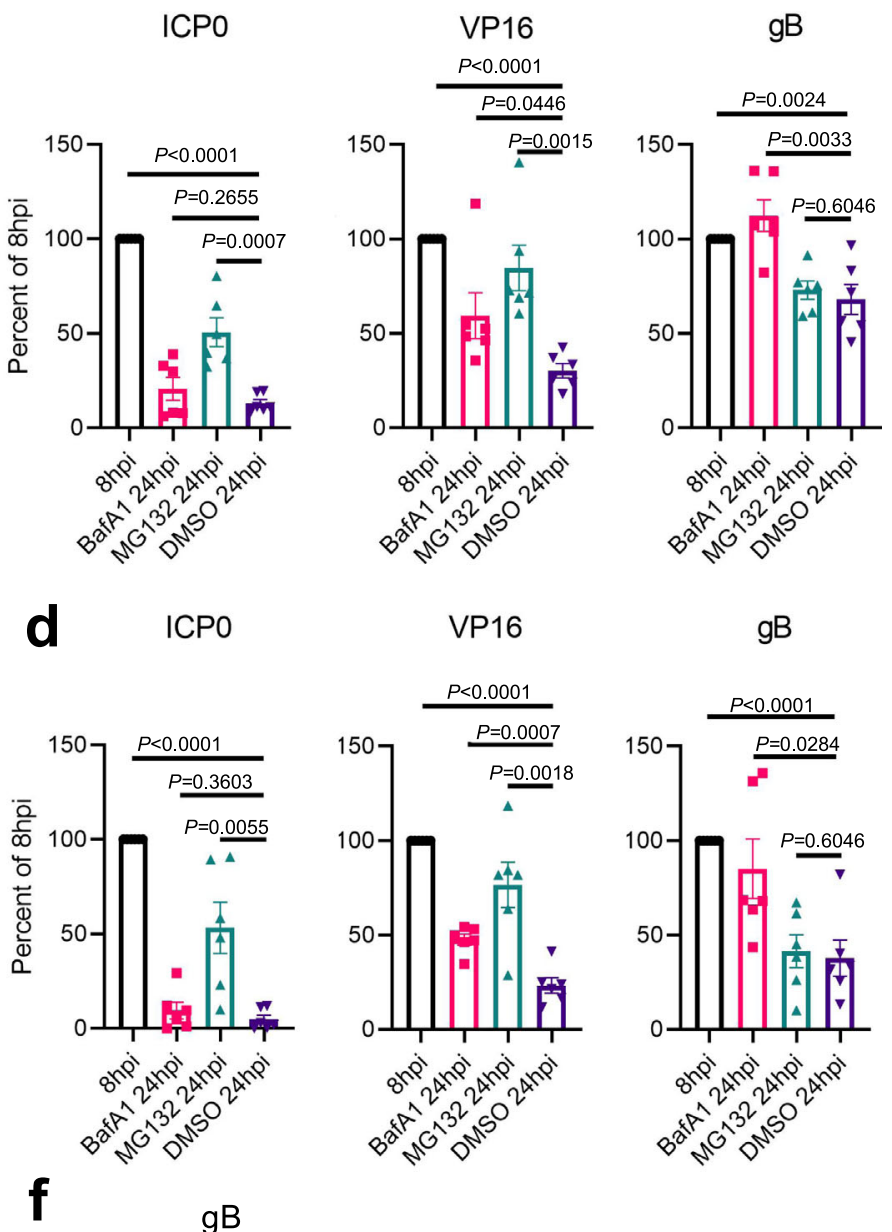

Fig. 3 HSV-1 VP16 and gB, but not ICPO, are degraded in an autophagy dependent manner. Wildtype cells were infected at 1 MOI with HSV-1 17-strain for $8 \mathrm{~h}$ before $\mathrm{CHX}$ addition to block protein synthesis. In combination with $\mathrm{CHX}$, either the autophagy inhibitor BafA1, the proteosome inhibitor MG132, or DMSO were added to cells. Cells were sampled at $8 \mathrm{~h}$, and $24 \mathrm{~h}$ after infection. a Shown for HeLa cells are immunoblots against the HSV-1 proteins gB, ICPO, VP16, and the internal reference GAPDH, b with band quantification relative to GAPDH ( $n=6$ replicates). c Shown for HCE cells are immunoblots against the HSV-1 proteins gB, ICPO, VP16, and the internal reference GAPDH, $\mathbf{d}$ with band quantification relative to GAPDH ( $n=6$ replicates). Similarly, LUHMES cells were infected at $2.5 \mathrm{MOI}$ with HSV-1 17-strain for $8 \mathrm{~h}$ before $\mathrm{CHX}$ addition to block protein synthesis. In combination with CHX, either BafA1, MG132 or DMSO were added to cells. Cells were sampled at $8 \mathrm{~h}$, and $24 \mathrm{~h}$ after infection. e Shown for LUHMES cells are immunoblots against the HSV-1 protein $\mathrm{gB}$ and the internal reference GAPDH, $\mathbf{f}$ with band quantification relative to GAPDH $(n=4$ replicates). Data are presented as mean values \pm SEM $(\mathbf{b}, \mathbf{d}, \mathbf{f})$. Two-tailed Student's $t$ test was performed for statistical analysis $(\alpha=0.05)$. ${ }^{*} p<0.05 ;{ }^{* *} p<0.01 ;{ }^{* * *} p<0.001,{ }^{* * * *} p<0.0001$, ns not significant. Source data are provided as a Source Data file.

expression was unaltered. Additionally, we observed that MG132 also resulted in significant inhibition of VP16 degradation, suggesting there may be multiple pathways involved in the degradation of VP16 during infection. $\mathrm{gB}$ is an essential glycoprotein present on the envelope of HSV-1 virions, and is responsible for fusion with host cell membranes upon entry ${ }^{30}$. VP16 is a transactivating factor that hijacks host transcription factors to promote transcription of viral genes during the lytic cycle of infection $^{31}$. This restricted the spread of HSV-1 and PRV in different cell lines.

In a report published during this study, OPTN was shown to be downregulated as a potential strategy to enhance HSV-1 

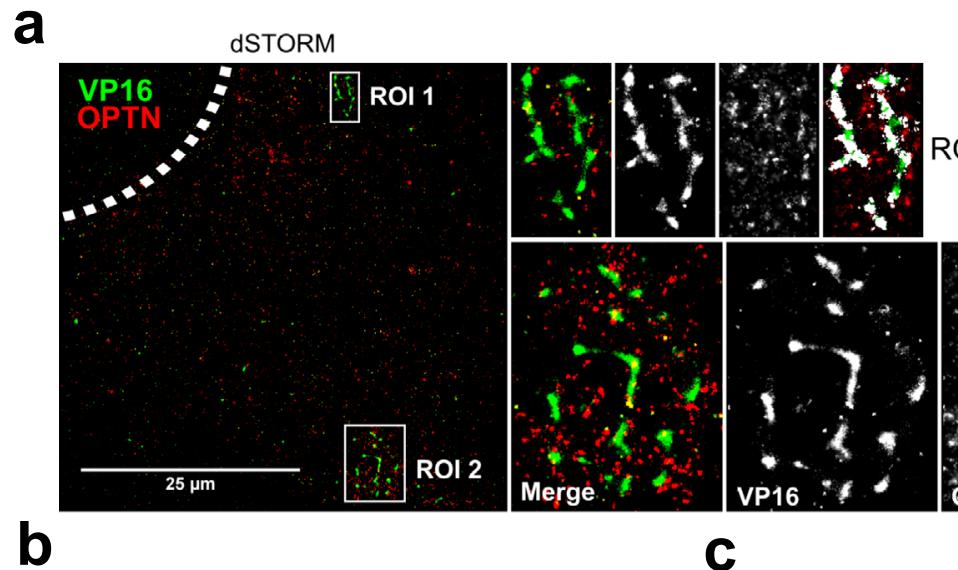
ROI 1
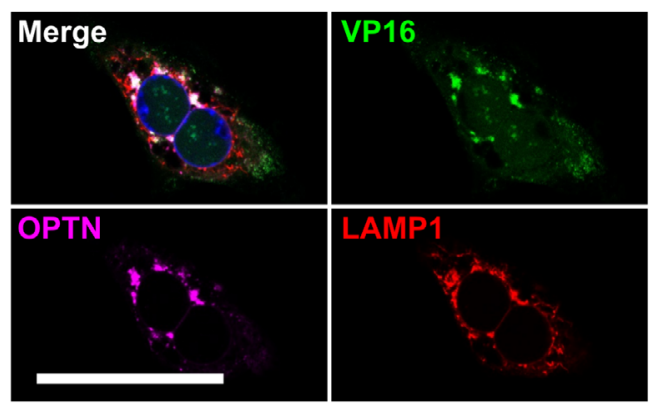

C
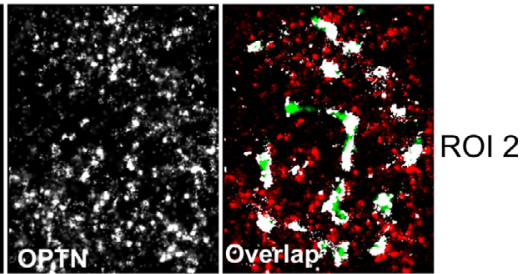

e
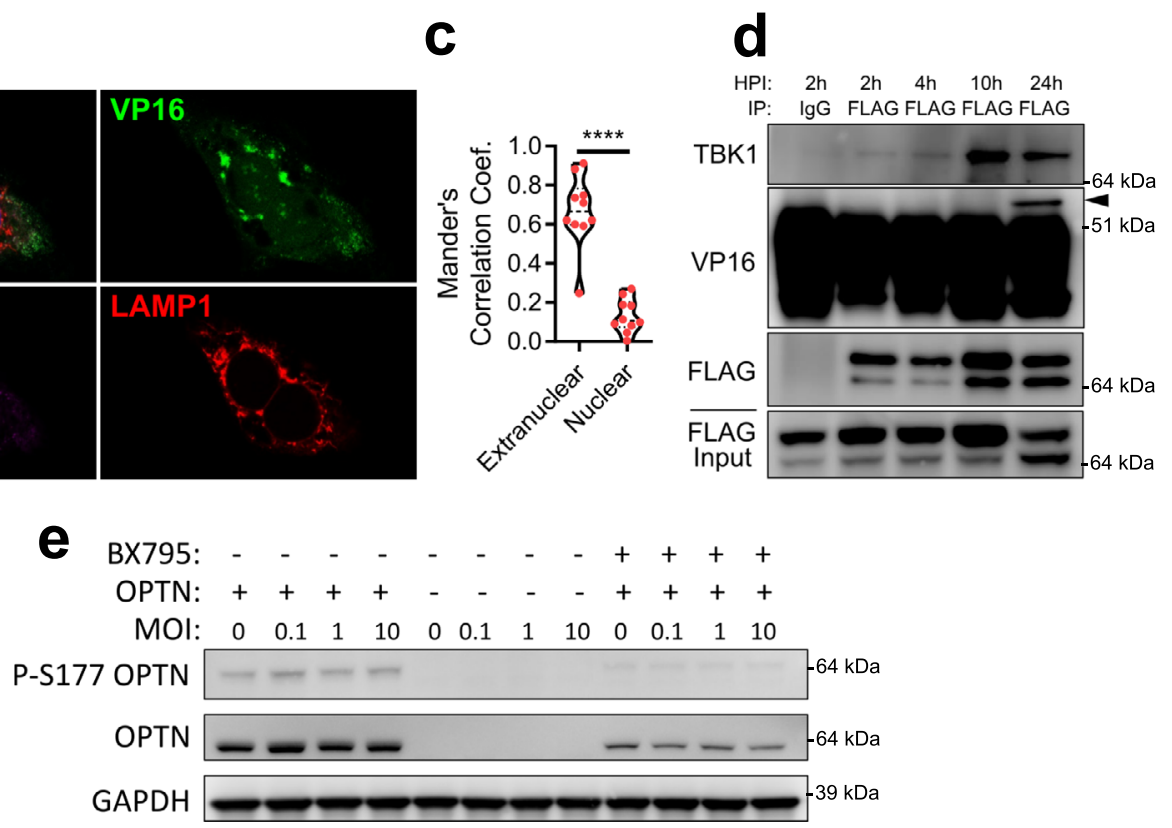

Fig. 4 OPTN is associated with VP16 during HSV-1 infection and is activated by TBK1. a dSTORM super-resolution microscopy for OPTN and VP16 staining. Enlarged images highlight association of OPTN and VP16 stained structures. Shown to the right are the enlarged images for regions of interest (ROI) representing the merged images, the raw signal for VP16 and OPTN, and the pixel overlap between the channels. Scale bar is $25 \mu$ m. Dashed line represents nucleus. $\mathbf{b}$ Representative image of Optn+/+ cells infected with VP16-gfp HSV-1 at MOI 1 for $12 \mathrm{~h}$ stained for OPTN and LAMP1. Scale bar is $50 \mu \mathrm{m}$. c Mander's colocalization coefficient representing the proportion of VP16 and OPTN co-staining. $n=10$ cells analyzed for colocalization. $P<0.0001$. d Immunoblot of anti-FLAG co-immunoprecipitation of Optn+/+ cells expressing FLAG-OPTN and infected with HSV-1 at 1 MOI. Arrow points to VP16 band. e Immunoblot of Optn+/+, Optn-/-, or Optn+/+ cells treated with TBK1 inhibitor, BX795 (50 $\mu$ M), infected with HSV-1 for $3 \mathrm{~h}$. Two-tailed Student's $t$ test was performed for statistical analysis $(\alpha=0.05) . * * * p<0.0001$. Source data are provided as a Source Data file.

infection, but OPTN knockdown did not result in increased HSV1 infection in human embryonic lung fibroblasts ${ }^{32}$. We have shown in HCE cells and LUHMES differentiated into neurons that OPTN knockdown leads to enhanced infection. Additionally, our in vitro and in vivo knockout models showed increased infection by HSV-1. This is the first demonstration that OPTNmediated autophagy is involved in degradation of HSV-1 particles and has an antiviral role. Interestingly, this is in contrast with the pro-viral role reported elsewhere for RNA viruses ${ }^{23}$. However, unlike our study, the RNA virus study did not use knockout in vitro and in vivo models to arrive at their conclusions. OPTN may have different effects in response to viruses ranging from restrictive to permissive depending on the lifecycle and hostinteractions of the virus in question. Additionally, other autophagy receptors may demonstrate similar anti-viral capabilities during HSV-1 infection.

Despite the role of OPTN in reducing HSV-1 protein expression and restricting spread of infection, OPTN does not affect the total level of autophagy within cells during infection of the knockdown or knockout in vitro models. Unexpectedly, the presence of OPTN had no effect on the level of replication of a $\gamma_{1} 34.5$ null HSV-1 strain. Regardless of starting protein level, cells were able to degrade VP16 and gB proteins upon cycloheximide addition and the total level of LC3 lipidation was unchanged. We interpret these observations to suggest that OPTN selective targeting of viral proteins is not crucial when $\gamma_{1} 34.5$ is unable to inhibit host autophagosome formation. This might be because there are potentially more available autophagosomes to compensate for lack of OPTN. Further study is required to better understand the interaction of $\gamma_{1} 34.5$ and OPTN.

Beyond degrading HSV-1 protein, we show that OPTN is closely linked with optimal immune function and neuronal survival. OPTN has been shown to regulate interferon induction, and TNFR (TNF- $\alpha$ receptor) signaling to the benefit or detriment of cells $^{21-23}$. Much previous work on OPTN-dependent regulation of cytokine signaling has been performed in vitro, but our in vivo data shows tissue specific differences in cytokine profiles between the Optn+I+ and $-/-$ mouse strains. In the dLNs, there was 

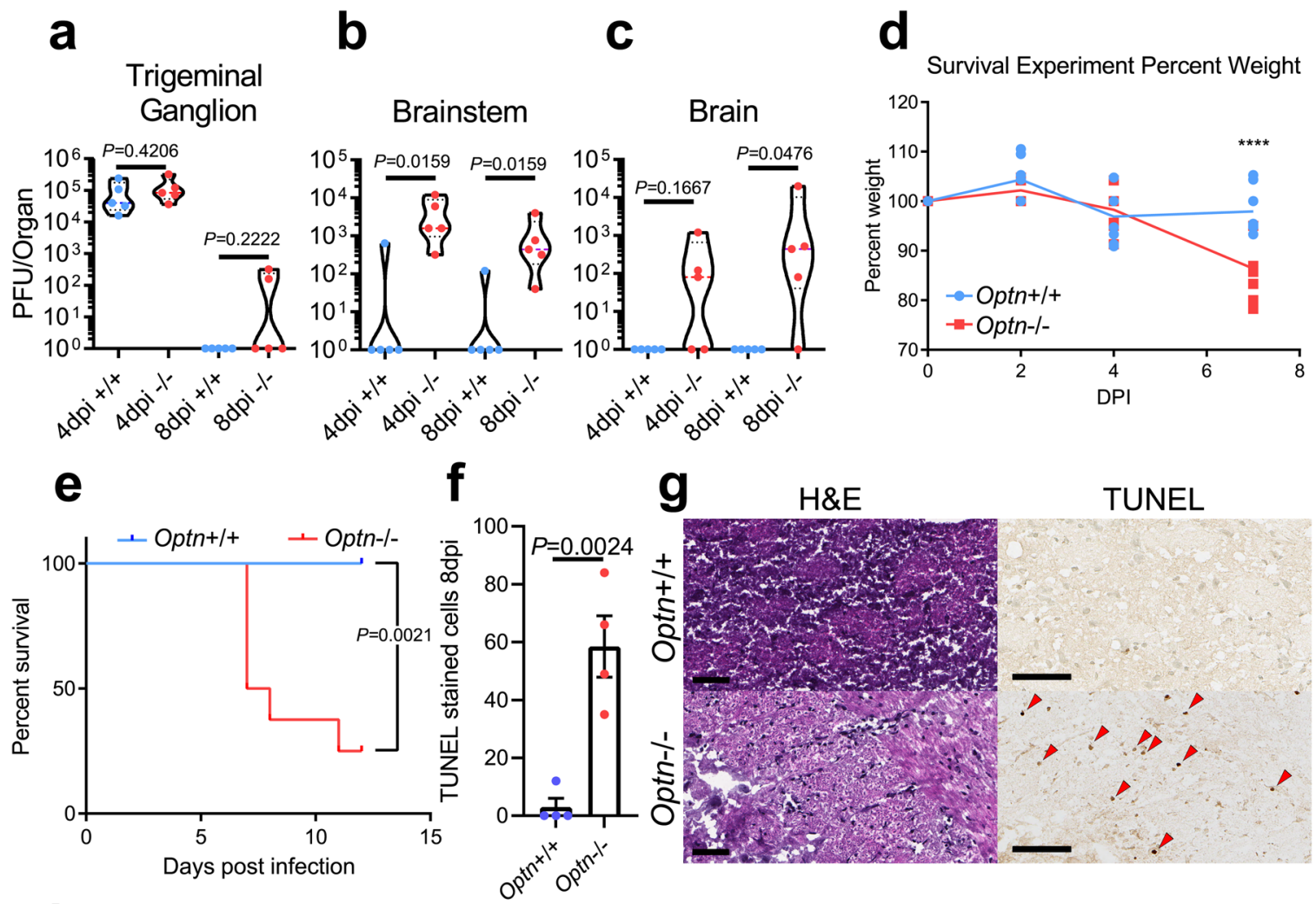

h

Control

ALS

HSE

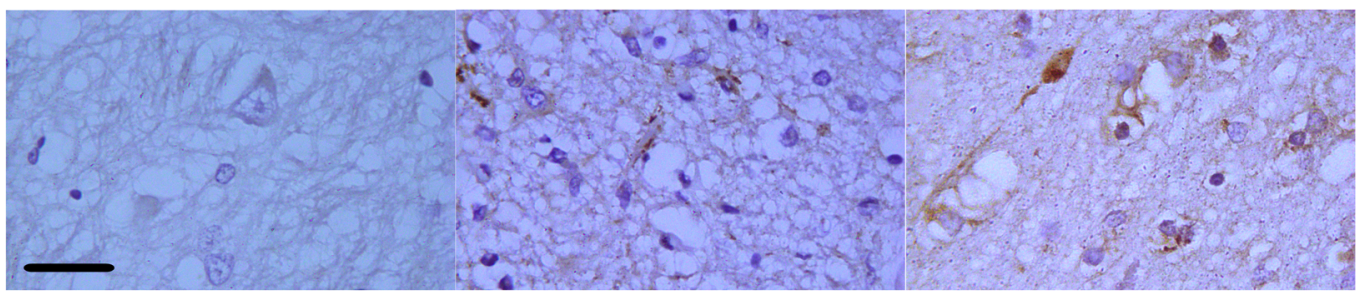

i

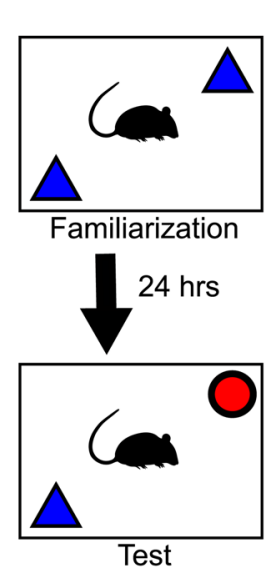

j

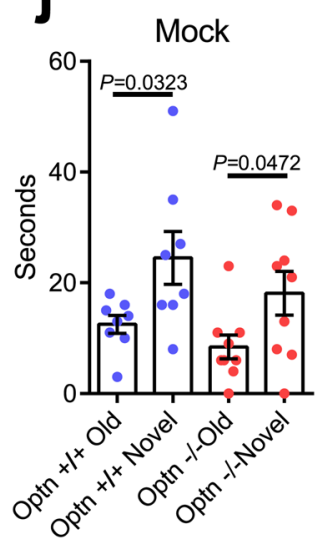

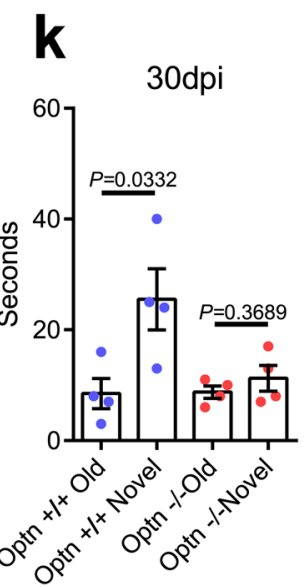

enhanced immune activation when OPTN was present, but the CNS tissue revealed the opposite trend where IFN- $\gamma$ was highly represented in OPTN-deficient mice. This shows there are further tissue specific determinants of cytokine signaling which interact with OPTN to drive or negate cytokine production such as cell types, local immunity, or permissiveness to HSV-1 infection, and these determinants are linked to selective autophagy ${ }^{21-23}$.

Downstream of TNFR stimulation, OPTN selectively degrades RIPK1, a necroptosis signaling molecule implicated in increased chronic CNS necroptosis ${ }^{24}$. Previous reports have implicated 
Fig. 5 OPTN is neuroprotective against herpes simplex virus encephalitis. Mice were infected using corneal scarification using $5 \times 10^{5} \mathrm{PFU}$ of $\mathrm{HSV}-1$ McKrae strain. a-c Tissue plaque assay for trigeminal ganglion (left), brainstem (middle), and brain (right) ( $n=5$ mice per group). Two-tailed Mann-Whitney $U$ test was used where $p<0.05$ indicates significance. $\mathbf{d}$ Weight changes of mice $(n=5$ mice per group) up to $7 \mathrm{dpi}$. Two-way repeatedmeasures ANOVA $F_{(3,42)}=9.908, p<0.0001$. e Kaplan-Meier curve for HSV-1 infected Optn+/+ $(n=8)$ and $-/-(n=8)$ mice. Logrank test was used to compare curves with $p<0.05$ being significant. $\mathbf{f}, \mathbf{g}$ (left) Quantitation of TUNEL stained cells in brainstems of Optn $+/+$ and $-/-$ mice $8 \mathrm{dpi}(n=4$ mice per group). (right) Representative images of H\&E staining and TUNEL staining (dark brown) for brainstems. Arrows highlight TUNEL positive cells. Scale bar is $50 \mu \mathrm{m}$. h Immunohistochemistry staining of OPTN (dark brown) in human brainstem tissue from a healthy, amyotrophic lateral sclerosis (ALS), or HSE patient shows aggregation of OPTN protein expression in diseased tissues. Scale bar is $100 \mu \mathrm{m}$. i Schematic of Novel Object Recognition (NOR) test for mice. j mock-infected Optn $+/+$ and $-/-$ mouse exploration times for old and novel objects. ( $n=8$ mice per group) (k) Optn $+/+$ and $-/-$ mouse exploration times for old and novel objects at $30 \mathrm{dpi}$ with $1 \times 10^{5}$ PFU of HSV-1 McKrae strain ( $n=4$ mice per group). Data are presented as mean values $\pm \operatorname{SEM}(\mathbf{f}, \mathbf{j}, \mathbf{k})$. Unless otherwise indicated, Two-tailed Student's $t$ test was performed for statistical analysis $(\alpha=0.05) .{ }^{*} p<0.05 ; * * p<0.01$; **** $p<0.0001$, ns not significant. Source data are provided as a Source Data file.

OPTN deficiency as a mechanism for the progression of $\mathrm{ALS}^{24}$. We have shown that acute CNS necroptosis can occur during HSV-1 infection in Optn-/- mice, leading to encephalitis, longterm neurodegeneration, and death, further supporting the antiviral restriction by OPTN. This is rescuable by treatment with the RIPK1 inhibitor, Nec1s. Nec1s may be a potential drug candidate to treat viral encephalitis, but the efficacy needs to be directly assessed before use outside of animal models.

Lastly this study focused on the role of OPTN in protection of the CNS, but the study has not addressed the role of OPTN in the peripheral nervous system (PNS). In both the Optn+I+ and $-1-$ mice the infection reached the trigeminal ganglion but failed to spread to the CNS of Optn+/+ mice. HSV-1 treatments and pathologies are complicated by the ability of herpesviruses to establish latency. OPTN suppresses the expression of VP16, a key protein in establishment and reactivation from latency. OPTN suppression of VP16 might prevent HSV-1 reactivation from latency because VP16 is a transactivating factor that can drive expression of a lytic gene program in sensory neurons ${ }^{33,34}$. Future work is required to investigate the relationship between OPTN and latency.

Selective autophagy has emerged as an important cellular process for maintaining the health of neuronal tissues and resisting neurodegeneration. Dysfunction in OPTN is implicated in glaucoma and neurodegenerative diseases including ALS and $\mathrm{AD}^{5,18}$. Viral infection of the CNS has been hypothesized to contribute to the etiology of neurodegenerative disorders, and defects in autophagy may synergize with infection and subclinical neuroinflammation to accelerate neurodegeneration ${ }^{1-3}$. We show that mice lacking OPTN demonstrate accelerated decline in cognitive functions following $\mathrm{HSV}-1$ infection, underscoring the role of OPTN and HSV-1 in the potential etiology of neurodegeneration with cognitive decline. Many viruses encode virulence factors that alter host autophagy machinery, and despite the rarity of viral encephalitis, many individuals are seropositive for viruses with this capability ${ }^{4}$. Taken together we have shown the necessity of OPTN-mediated selective autophagy in targeting of HSV-1, recruitment of wider immune responses, and protecting the CNS from neurotropic viral infection and neuronal cell death. Our study demonstrates that defects in OPTN functions compounded by HSV-1 infection have the potential to cause accelerated neuronal damage.

\section{Methods}

Antibodies, stains, cells, viruses, chemicals, and plasmids. The following antibodies and stains were used in this study for imaging:

DAPI (D9542, Sigma) (1:1000), NucBlue ${ }^{\mathrm{Tx}}$ Live ReadyProbes ${ }^{\mathrm{Tx}}$ Reagent (Thermo Fisher, R37605) (2 drops per ml), Mouse monoclonal to LAMP1 (Abcam, ab25630, [H4A3]) (1:100), Mouse monoclonal anti-HSV1 + HSV2 VP16 (Abcam, ab110226, [LP1]) (1:100), Goat anti-Mouse IgG $(\mathrm{H}+\mathrm{L})$ Highly Cross-Adsorbed Secondary Antibody, Alexa Fluor 546 (Thermo Fisher, A-11030) (1:100), Goat anti-Rabbit IgG $(\mathrm{H}+\mathrm{L})$ Highly Cross-Adsorbed Secondary Antibody, Alexa Fluor 647
(Thermo Fisher, A-21245) (1:100), Rabbit polyclonal anti- Optineurin (CTerm) (Cayman Chemical, No. 100000) (1:100), and anti-mouse CD8a APC-conjugated monoclonal antibody (Tonbo biosciences, 20-1886-U100, [clone 2.43]) (1:100).

The following antibodies were used for immunoblot:

Mouse monoclonal anti-GAPDH (Santa Cruz, sc-69778, [7B]) (1:1000), Goat anti-Mouse IgG $(\mathrm{H}+\mathrm{L})$ Highly Cross-Adsorbed Secondary Antibody HRP (Thermo Fisher, 31432) (1:5000), Goat anti-Rabbit IgG (H L) Cross Adsorbed Secondary Antibody HRP (Thermo Fisher, G-21234) (1:5000), Mouse monoclonal anti-FLAG (Sigma, F1804, [M2]) (1:1000), Mouse monoclonal anti-HSV1 ICP0 (Abcam, ab6513, [5H7]) (1:1000), Rabbit monoclonal anti-p-S177 OPTN (Cell Signaling Technologies, 57548 S) (1:1000), Mouse monoclonal anti-HSV1 + HSV2 VP16 (Abcam, ab110226, [LP1]) (1:1000), and Rabbit polyclonal anti- Optineurin (CTerm) (Cayman Chemical, No. 100000) (1:1000).

The following antibodies were used for immunoprecipitation:

Mouse monoclonal anti-FLAG (Sigma, F1804, [M2]) $(5 \mu \mathrm{g})$, normal mouse IgG (Santa Cruz, sc-2025) (5 $\mu \mathrm{g}$ ), and additionally, and Protein A/G PLUSAgarose beads (Santa Cruz, sc-2003) $(20 \mu \mathrm{l})$ were used.

For flow cytometry the following antibodies were used:

anti-mouse CD8a APC-conjugated monoclonal antibody (Tonbo biosciences, 20-1886-U100, [clone 2.43]) (1:100). anti-mouse CD4 PE-conjugated monoclonal antibody (Tonbo biosciences, 50-0042-U100, [RM 4-5]) (1:100).

HeLa Optn-/ - and parental strain were provided by Dr. Richard Youle (National Institutes of Health). Vero cells (ATCC) were used in plaque assays and in virus preparation. LUHMES cells (ATCC) were provided by Dr. David Bloom (University of Florida). HCE cells were provided by Dr. Kozaburo Hayashi (National Eye Institute, Bethesda, MD). Paul Kinchington (University of Pittsburgh) provided the dual tagged KOS strain of HSV-1 (RFP driven by gC promoter and GFP driven by ICP0 promoter).

Dr. Patricia Spear (Northwestern University) provided the KOS and 17 strains The strain $17 \gamma_{1} 34.5$-null mutant of HSV-1 was provided by Dr. David Leib (Dartmouth College). Dr. Prashant Desai (Johns Hopkins University) provided the HSV-1 K26-GFP KOS strain. Dr. Steven Triezenberg (Van Andel Research Institute) provided the DG1 (VP16-GFP expressing) strain of HSV-1. Gregory Smith (Northwestern University) provided the PRV-GS2484 strain (PRV-Becker expressing mRFP1-UL35 fusion protein and CMV-GFP cassette in Us4).

The following chemicals were used in this study: Bafilomycin A1 (Sigma) to inhibit autophagic flux, MG132 (Sigma) to inhibit proteasomal degradation. Cycloheximide (Sigma) to block de novo protein synthesis. Lipofectamine 2000 (Thermo Fisher) and RNAiMAX (Thermo Fisher) for transfections. Necrostatin-1s (Selleckchem) for inhibition of necroptosis. BX795 (Selleckchem) for inhibition of TBK1.

The FLAG-OPTN plasmid was provided by Dr. Beatrice Yue (University of Illinois at Chicago).

Mice. Mice used in this study were generated in the study ${ }^{35}$. In summary Optn $n^{\text {flox/ }}$ flox mice on a C57BL/6 background were generated using a targeting vectorinserted LoxP site that flanks the first coding exon and a neomycin selection cassette. Optn flox/flox mice were crossed with CMV-Cre mice (Jackson Laboratories, Bar Harbor, ME, USA) to generate CMV-Cre; Optn flox/wt mice, which were used as breeding pairs. Global Optn knockout (CMV-Cre; Optn flox/flox $)$ mice are hereafter referred to as Optn - /- mice. 8-16 weeks old Optn $-1-$ or Optn $+/+$ male and female mice were used in this study.

All experiments were performed and housed in a BSL2 rodent facility in the Biologic Resources Laboratory at the University of Illinois at Chicago with a standard $12 \mathrm{~h}$ light, $12 \mathrm{~h}$ dark cycle. Ambient temperatures at this facility are maintained between 20 and $26^{\circ} \mathrm{C}$ and relative humidity between $45 \%$ and $65 \%$. This modern animal facility has several veterinarians on staff available for expert veterinary care and advice during the project. Animal services core facility at the Department of Ophthalmology and visual sciences houses a BSL-2 facility dedicated specifically to our laboratory use. All protocols have been reviewed and certified by the animal care committee of the University of Illinois at Chicago. 
a
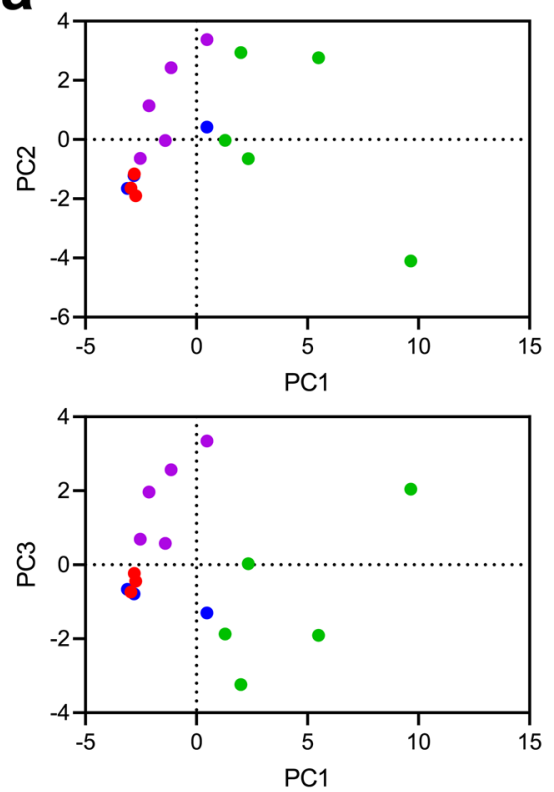

b
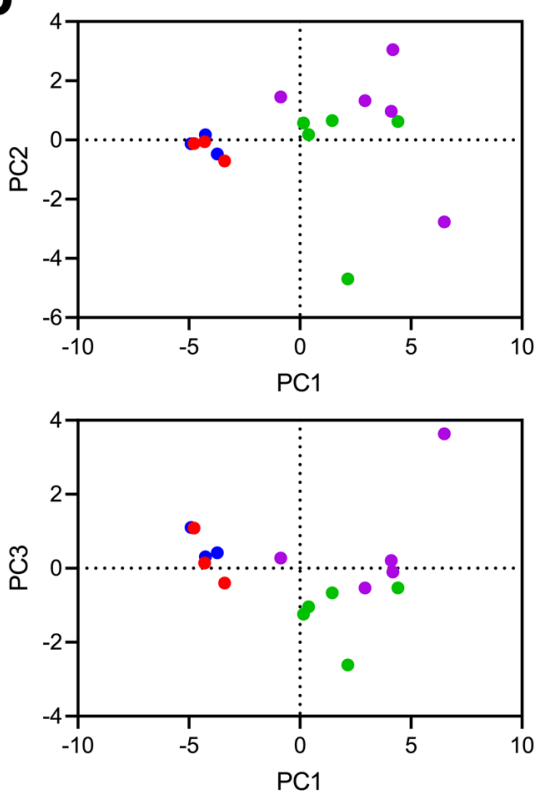

Group

Mock Optn+/+

Mock Optn-/-

8dpi Optn+/+

8dpi Optn-/-
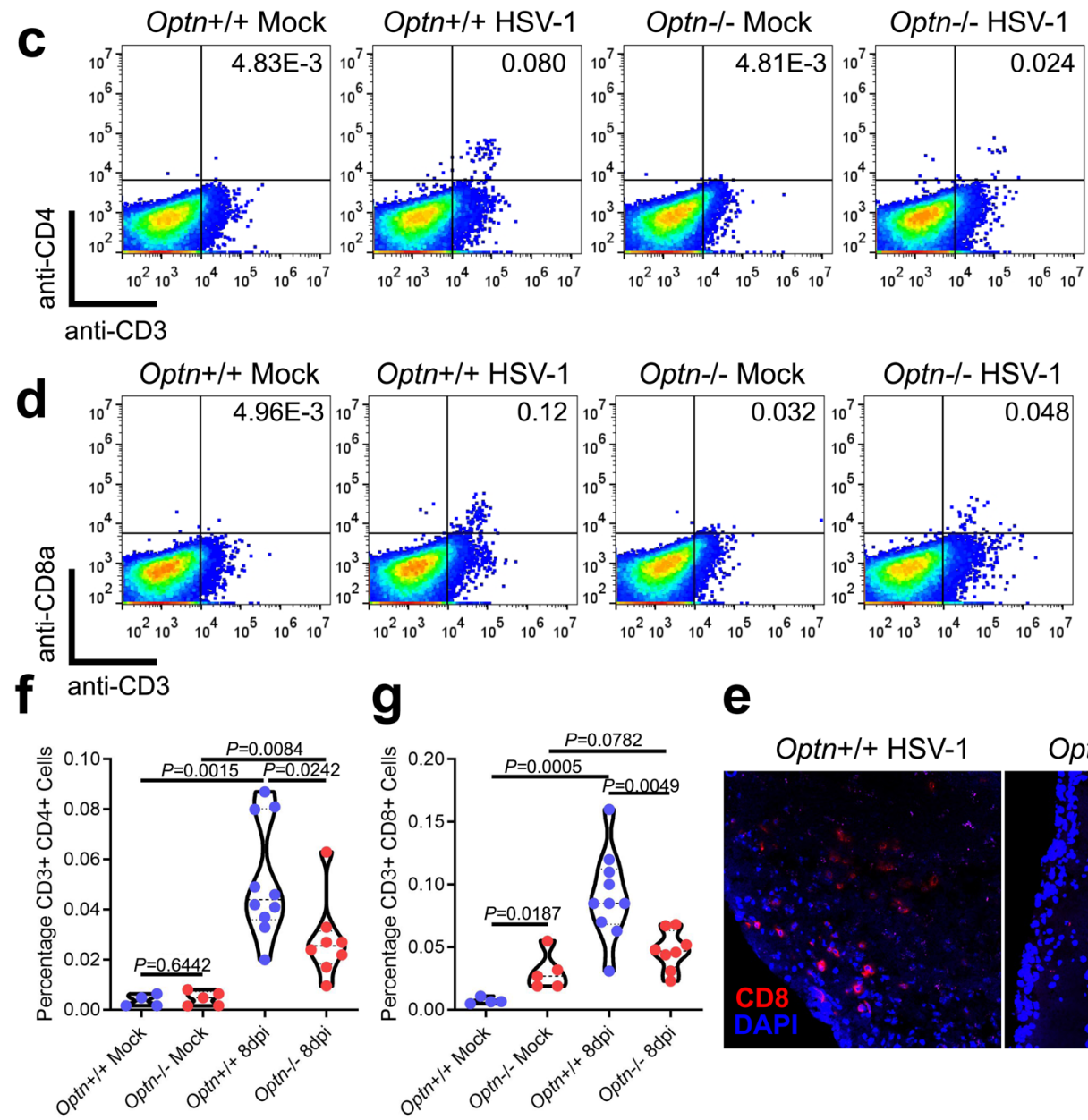

Optn+/+ HSV-1

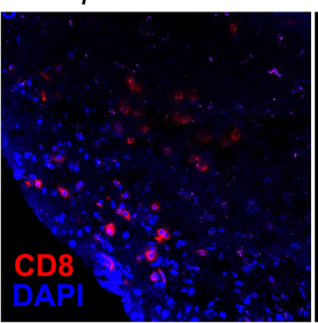

Optn-/- HSV-1

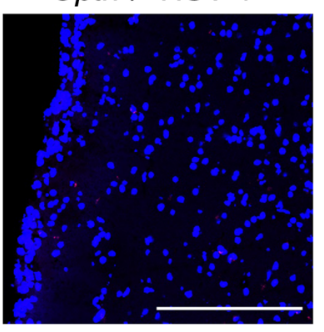

Fig. 6 OPTN deficiency impairs recruitment of CD4 and CD8 lymphocytes to the CNS. Principal component analysis of cytokine protein expression profiles from $8 \mathrm{dpi}$ ( $n=5$ mice per group) or mock infected ( $n=3$ mice per group) Optn $+/+$ or $-/-$ mice to compare (top) PC1 vs PC2 or (bottom) PC1 vs PC3 are shown for a draining lymph nodes and $\mathbf{b}$ brainstems. Brainstem cell suspensions from mock infected Optn $+/+(n=4$ mice), mock infected Optn $-/-(n=5$ mice $), 8 \mathrm{dpi}$ Optn $+/+(n=10$ mice $)$, and $8 \mathrm{dpi}$ Optn $-/-(n=8$ mice $)$ were analyzed using flow cytometry to measure the percentage of T cells present. c Dot plots representing CD3 + CD4 + cells. d Dot plots representing CD3 + CD $8+$ cells. $\mathbf{f}$ Quantification of percentage CD3 + CD4 + cells. $\mathbf{g}$ Quantification of CD3 + CD8 + cells. e Staining for CD8 in 8 dpi mouse brainstem tissue. Scale bar is $200 \mu$ m. Two-tailed Student's $t$ test was performed for statistical analysis $(\alpha=0.05) .{ }^{*} p<0.05 ;{ }^{* *} p<0.01 ;{ }^{* *} p<0.001$, ns, not significant. Source data are provided as a Source Data file. 
a
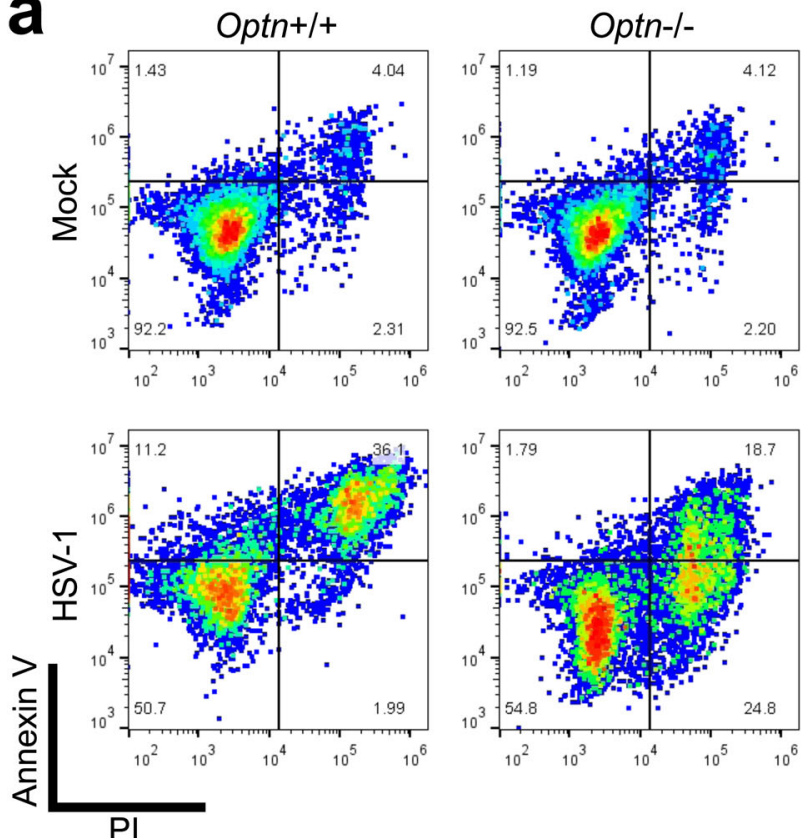

b
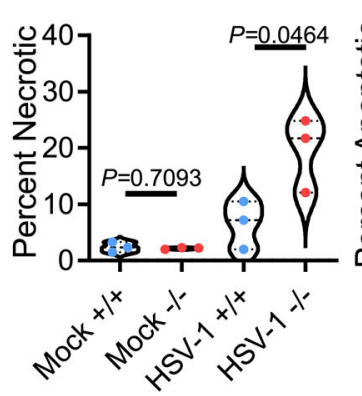

g
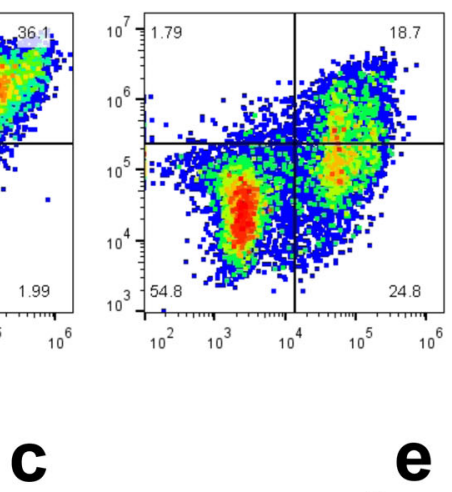

d

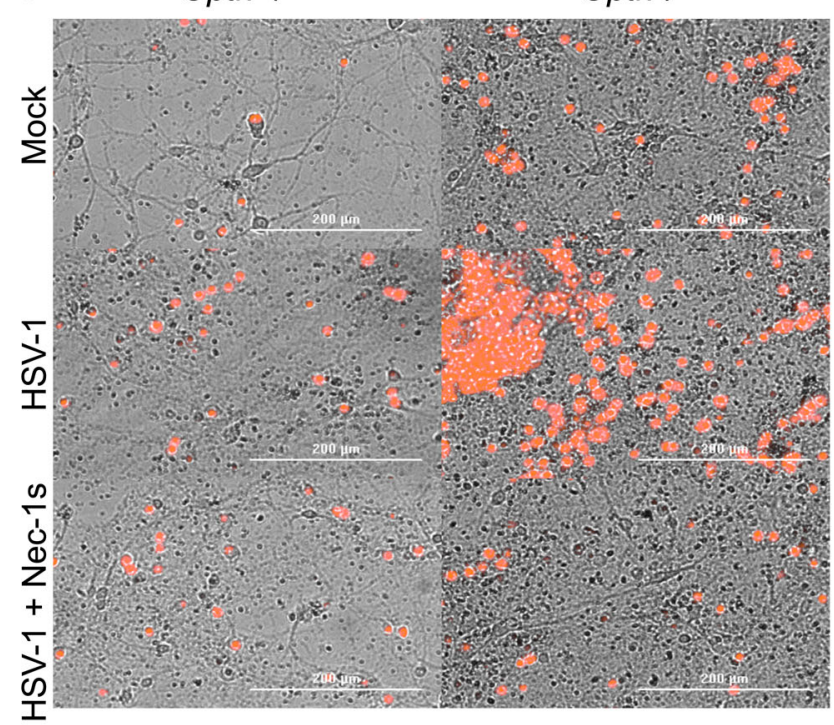

Optn-/-

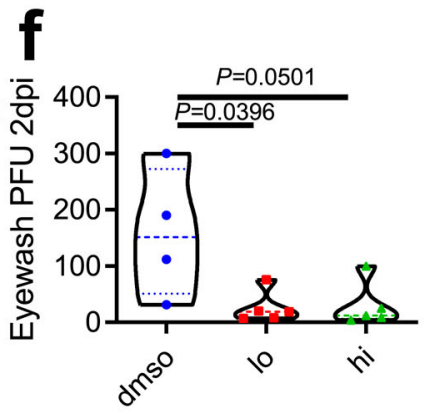

T
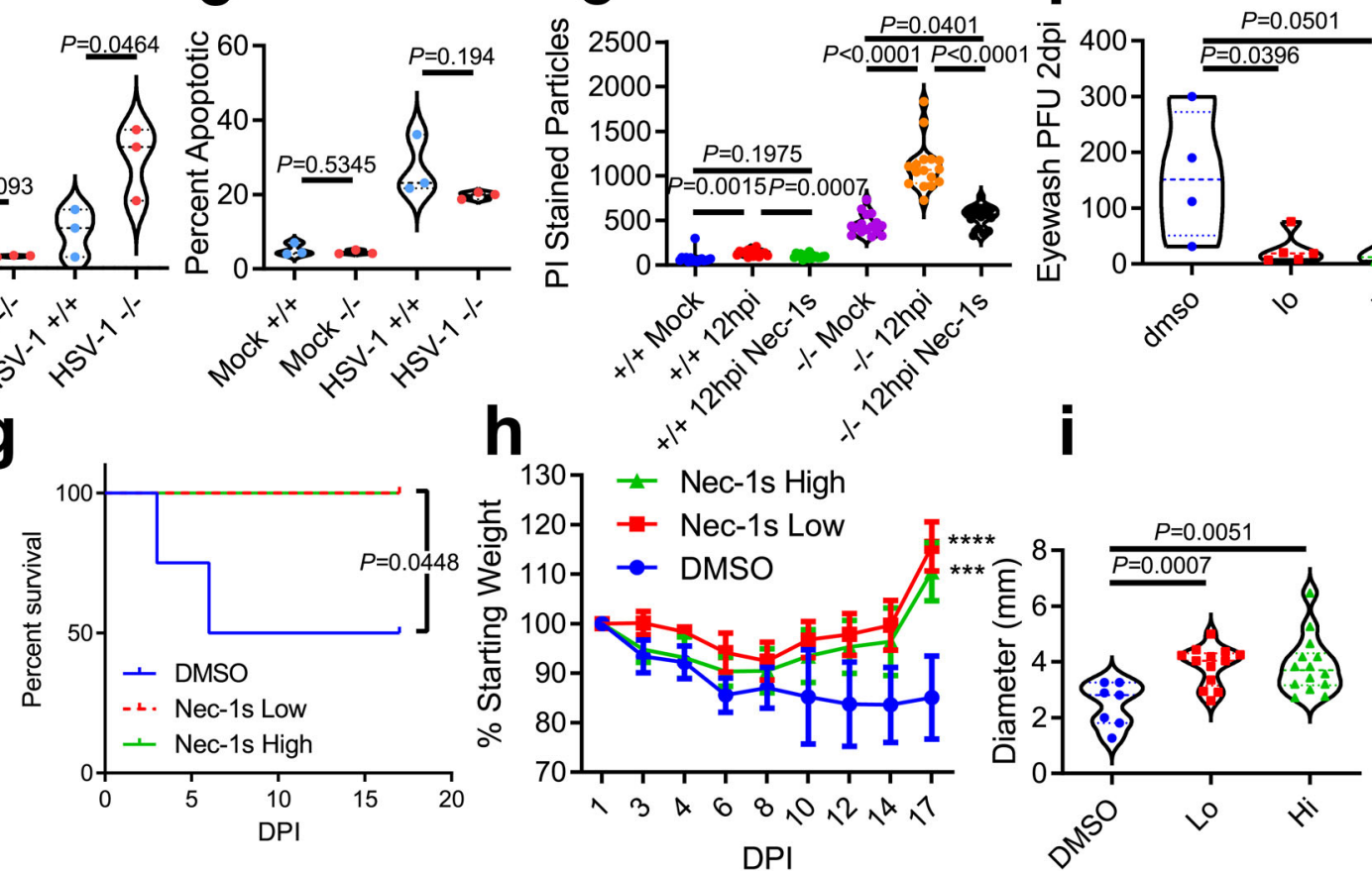

$\mathbf{1}$

Fig. 7 OPTN suppresses necroptosis in vitro and in neurons during HSV-1 infection. a Dot plots of flow cytometry analysis of Optn $+/+$ and $-/-$ cells stained for Annexin $\mathrm{V}$ and propidium iodide (PI). b, c Percentage of (b) Annexin $\mathrm{V}$ lo, PI hi cells indicating necrotic cells or c Annexin $\mathrm{V}$ hi, PI hi cells indicating apoptotic cells. d Representative images of primary embryonic neurons from Optn $+/+$ or $-/-$ mice that were mock infected, HSV-1 infected, or HSV-1 infected and Nec-1s treated. Dead cells were stained using PI (Red). e Image quantification of PI-stained primary neurons. f-i Optn-/- were treated with hi Nec-1s ( $n=5$ mice), lo Nec-1s $(n=5$ mice), or DMSO ( $n=4$ mice) during infection. f Plaque assay on ocular washes from Optn-/ - mice 2 dpi. $\mathbf{g}$ Kaplan-Meier curve for HSV-1 infected Optn-/- treated with DMSO, lo or hi Nec-1s. Logrank test for trend was used to compare curves with $P<0.05$ being significant. $\mathbf{h}$ Percentage of initial weight over time. Two-way repeated-measures ANOVA $F_{(16,88)}=1.758, p=0.0503$, multiple comparisons at 17 dpi (DMSO vs. Nec-1s Low) $p<0.0001$, (DMSO vs. Nec-1s High) $p=0.0009$. i dLN diameters for Optn $-/-$ mice treated with DMSO, lo Nec-1s, or hi Nec$1 \mathrm{~s}$ at $17 \mathrm{dpi}$ and data are presented as mean values \pm SEM. Unless otherwise stated Two-tailed Student's $t$ test was performed for statistical analysis $(\alpha=0.05) .{ }^{*} p<0.05 ;{ }^{* *} p<0.01 ; * * p<0.001,{ }^{* * * *} p<0.0001$, ns not significant. Source data are provided as a Source Data file.

Mouse infection experiments. The corneal scarification method was used for infection of mice. Mice were anesthetized using a mixture of ketamine and xylazine, and the topical anesthetic, proparacaine, was applied to the ocular surface prior to epithelial debridement. Three parallel scratches were made in the corneal epithelium with a 30 -gauge needle, then mice were infected with $5 \times 10^{5} \mathrm{PFU}$ HSV1 by application of a virus containing solution to the corneal surface. For the novel object recognition test mice were infected with $1 \times 10^{5}$ PFU HSV-1 to reduced number of mice that reached endpoint criteria before $30 \mathrm{dpi}$. McKrae strain was 
used for mouse experiments. For survivorship endpoint criteria, mice had to lose $>15 \%$ initial body weight rapidly, demonstrate excessive morbidity, difficulty ambulating, paralysis, or deep lesions on the head $>1 \mathrm{~cm}^{2}$.

Novel Object Recognition Test. The Novel Object Recognition Test was be used to assess whether deterioration of memory is occurring in mice during ocular HSV1 infection. Mice were 8-16 weeks old. The apparatus for testing was a white polypropylene box with a video recording camera (GoPro Hero7, GoPro) mounted above it. The base of the box is 15 inches by 20 inches and the walls of the box are $11.75^{\prime \prime}$ inches high. The test for each treatment group included a familiarization session and a testing session, both of which were recorded.

The familiarization session began with cleaning the box and objects with MB10 to both disinfect the area and minimize odor cues. Each animal was subjected to a familiarization session where two identical objects were placed in the box prior to introducing the mouse to the box. The mouse was introduced to the box and allowed to explore the box for a total of $5 \mathrm{~min}$. At this point the mouse was removed from the testing area and the area and objects were cleaned again with MB10 before testing the next mouse.

$24 \mathrm{~h}$ later each animal was subjected to a testing session where two objects were placed in the box prior to introducing the mouse to the box. One object was from the familiarization session, and the second was a novel object. The session began with cleaning the box and objects with MB10 to both disinfect the area and minimize odor cues. The mouse was introduced to the box and allowed to explore the box for a total of $5 \mathrm{~min}$. At this point the mouse was removed from the testing area and the area was cleaned again with MB10 before testing the next mouse.

Using the recorded footage, the time the mouse explored each object was measured manually using a stopwatch. Only exploration including closely sniffing or touching the object with whiskers or nose was counted as exploration.

Necrostatin-1s experiments. For mouse experiments, Nec-1s was administered at a low dose of $0.5 \mathrm{mg} / \mathrm{kg}$ (lo), high dose of $2.5 \mathrm{mg} / \mathrm{kg}$ (hi), or control treatment of vehicle alone (DMSO). Administration by intraperitoneal injection was performed $12 \mathrm{~h}$ before infection (0dpi), then daily until $7 \mathrm{dpi}$. For in vitro experiments 15 days in vitro (DIV) primary neuron culture from Optn+1+ or Optn-1- mouse embryos were infected with MOI 2.517 strain HSV-1 for $1 \mathrm{~h}$ before medium was changed. The replacement medium included $1 \mu \mathrm{g} / \mathrm{ml}$ propidium iodide (Invitrogen) and either $100 \mu \mathrm{g} / \mathrm{mL} \mathrm{Nec-1s}$ or DMSO. Medium was used for the remainder of the experiment and images were captured using a Lionheart LX automated microscope.

BX795 experiments. $10^{6} \mathrm{HeLa} O p t n+/+$ or $-/$ - cells were plated in six-well format. The next day one group of wells were treated with $50 \mu \mathrm{M}$ BX795 for $1 \mathrm{~h}$ then all groups were infected at indicated MOI with 17 strain HSV-1 for $3 \mathrm{~h}$. Samples were harvested for immunoblotting.

Cycloheximide chase experiments. $10^{6} \mathrm{HeLa} O p t n+/+$ or $-/-$ cells were plated in six-well format, or $5 \times 10^{5} \mathrm{HCE}$ cells were plated in 12-well format and transfected with siRNA. The next day cells were infected at MOI 1 with HSV-1 (17strain) for $12 \mathrm{~h}$. Medium (DMEM 10\% FBS for HeLa, MEM 10\% FBS for HCE) was replaced with fresh medium containing $100 \mu \mathrm{g} / \mathrm{mL}$ cycloheximide to block de novo protein translation. Samples were collected at $12 \mathrm{~h}, 18 \mathrm{~h}$, and $24 \mathrm{~h}$ after infection for lysis in RIPA buffer and subsequent immunoblotting. In a separate experiment using only wildtype HeLa, LUHMES, or HCE cells, at 8 hpi fresh medium containing $100 \mu \mathrm{g} / \mathrm{ml}$ cycloheximide to block de novo protein translation and either DMSO, as a control, $200 \mathrm{nM}$ Bafilomycin A1 to inhibit autophagy, or $50 \mu \mathrm{M}$ MG132 to inhibit the proteasome. An untreated sample was taken at $8 \mathrm{hpi}$, and treated samples were taken at $24 \mathrm{hpi}$ for immunoblotting.

siRNA transfection. A Dicer-Substrate Short Interfering RNAs (DsiRNAs) Tri$\mathrm{FECTa}^{\circledast}$ Kit (IDT) with predesigned siRNA molecules was used for transfections in this study. Cells were plated and grown to $50 \%$ confluency. Cells were then transfected as per manufacturer's protocol using RNAiMAX at $1 \mu \mathrm{l} / \mathrm{mL}$ in OptiMEM (ThermoFisher). Multiple concentrations for each premade siRNA molecule were tested and it was determined that siRNA 1 at $1 \mathrm{nM}$ produced effective knockdown with minimal cell death after $48 \mathrm{~h}$ of transfection.

LUHMES cell culture. LUHMES cell culturing was adapted from the methods outlined ${ }^{36}$. First tissue culture flasks and plates were coated with poly-l-ornithine hydrobromide (Sigma) overnight in a biosafety cabinet then with fibronectin (Sigma) overnight in a tissue culture incubator at $37^{\circ} \mathrm{C}$. Plates were allowed to air dry before use. Proliferation medium (DMEM:F12 (ATCC) containing 1\% N2 Supplement (ThermoFisher), 1X Penicillin-Streptomycin-Glutamine solution (ThermoFisher), and recombinant human FGF-basic (Fibroblast Growth Factor, Peprotech) was prepared immediately before use and was used to passage the undifferentiated LUHMES cells. Cells were plated in plates and grown to $50 \%$ confluency before differentiating in differentiation medium with $1 \mu \mathrm{g} / \mathrm{mL}$ tetra-

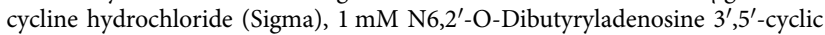
monophosphate sodium salt (Sigma), and $2 \mathrm{ng} / \mathrm{mL}$ Glial cell-derived neurotrophic factor (GDNF). In experiments where siRNA transfection was required, the transfection was performed overnight in OptiMEM (see siRNA transfection section) prior to addition of differentiation medium. Cells were then used for experiments 4 days after differentiation.

Primary neuron culture. Embryonic brains from E18 mouse embryos were pooled for each mouse strain and digested in $5 \mathrm{~mL} 0.25 \%$ Trypsin with $75 \mu \mathrm{L} 0.1 \%$ DNase (New England Biolabs) in a $37^{\circ} \mathrm{C}$ water bath for $30 \mathrm{~min}$. Tissue was spun down at $500 \times g$ for $1 \mathrm{~min}$ to aspirate trypsin and wash pellet with $5 \mathrm{~mL} \mathrm{cFBS}$ (Gibco). Cells were spun down and resuspended in $5 \mathrm{~mL}$ medium $\left(485 \mathrm{ml} \mathrm{Neurobasal}{ }^{\circledR}\right.$-A medium (Gibco), $10 \mathrm{~mL} \mathrm{B27}$ supplement (Invitrogen), $5 \mathrm{~mL} 200 \mathrm{mM}$ L-glutamine (Invitrogen), $25 \mathrm{mM}$ glucose). Tissue was triturated with a $5 \mathrm{~mL}$ plastic pipette for 10 repetitions, then with a $1000 \mu \mathrm{L}$ pipette for 10 repetitions before being filtered through a $70 \mu$ strainer into a new $50 \mathrm{~mL}$ conical tube and spun down. The medium was aspirated and the cells were resuspended in $20 \mathrm{~mL}$ medium. In all, $0.5 \times 10^{6}$ cells were plated in $1 \mathrm{~mL}$ medium into Poly-D-Lysine coated 24-well plates. At 3 DIV a half medium change with Cytosine $\beta$-D-arabinofuranoside hydrochloride (Ara-C) containing medium to a final concentration of $1 \mu \mathrm{M}$ Ara-C. Subsequent restorative half medium without Ara-C were performed at DIV6, DIV8, DIV10, and DIV13. Experiments were performed on DIV15.

Viral genome isolation. Infected cell pellets were suspended in buffer containing $1 \%$ SDS, $50 \mathrm{mM}$ Tris (pH 7.5), and $10 \mathrm{mM}$ EDTA, and proteinase $\mathrm{K}$ ( 2 units $/ \mathrm{mL}$ ) then incubated overnight at $45^{\circ} \mathrm{C}$. Proteinase $\mathrm{K}$ is then heat inactivated for $30 \mathrm{~min}$ at $95^{\circ} \mathrm{C}$. DNA was isolated using phenol/chloroform extraction followed by ethanol precipitation. HSV-1 genomes were quantified using quantitative PCR (ABI 7500, Applied Biosystems) using HSV-1 specific primers and Fast SYBR Green Master Mix (Life Technologies). Primers are listed in Supplementary Table 1.

Time-lapse fluorescent microscopy. Immediately after the addition of HSV-1 in cell growth medium (DMEM, 10\% FBS, 1\% Penn/Strep), cells were placed in the environmentally controlled chamber on the stage of a Zeiss Observer spinning disk confocal microscope set to $37^{\circ} \mathrm{C}$ and $5 \% \mathrm{CO}_{2}$. Time-lapse experiments images were taken at $30 \mathrm{~min}$ intervals or $60 \mathrm{~min}$ intervals. Imaging was performed using either $10 \mathrm{x}$ or $100 \mathrm{x}$ objective lenses. Image analyses were performed in ZEN and imageJ software.

Fluorescent microscopy. Cells grown on $1.5 \mathrm{~mm}$ thick glass bottom dishes were fixed in $4 \%$ paraformaldehyde for $10 \mathrm{~min}$. If antibody staining was performed, fixation was followed by permeabilization in a $0.1 \%$ Triton X 100 solution. Samples were blocked in 5\% fetal bovine serum in phosphate-buffered saline (blocking buffer) for $1 \mathrm{~h}$. Samples were stained with primary antibody (1:100) in blocking buffer for $1 \mathrm{~h}$ followed by secondary antibody (1:100) and DAPI staining for $1 \mathrm{~h}$ Images were taken on a Zeiss LSM710 confocal microscope using 63x or 100x objective lenses. For the mouse brain sections, additional images were captured on a BioTek Lionheart microscope at $\times 10$ and image-stitched using the Biotek GEN5 v3.04 Imager software.

TIRF/super-resolution microscopy. Following the same staining protocol for fluorescent microscopy with two modifications; cells were washed five times with blocking buffer after each staining, and antibody concentrations were doubled (1:50) with DAPI omitted. Imaging was performed using a DMi8 S platform equipped for TIRF and ground state depletion (GSD) (Leica). OxEA buffer, an oxygen scavenger buffer, must be made immediately before use and is exhausted after $\sim 1 \mathrm{~h}$ of imaging. OxEA (50 mM $\beta$-MercaptoEthylamine hydrochloride [Sigma], 3\% v/v OxyFluor [Oxyrase Inc], 20\% v/v sodium DL-lactate solution [Sigma], in PBS pH 8-8.5 adjust with $\mathrm{NaOH}$ ) was added to the fixed, permeabilized, and stained cells prior to imaging. Once a location of interest was TIRFimaged using the LAS X software to calculate and adjust the machine for a $250 \mathrm{~nm}$ depth image, GSD was used starting with the longest excitation wavelength and ending with the shortest to collect 5000 images of "blinking" fluorescence for each channel. Post-imaging dSTORM reconstruction in FIJI with the ThunderSTORM plugin was performed to acquire super-resolution images.

Immunohistochemistry. Tissue was embedded in OCT Compound (Thermo Fisher Scientific) and frozen. $10 \mu \mathrm{m}$ sections were cut from the tissue in a CryoStar NX50 (Thermo Fisher Scientific) cryotome and collected on double frosted slides (Thermo Fisher Scientific). Slides were fixed in cold 100\% acetone for $10 \mathrm{~min}$. Slides are then washed three times in phosphate-buffered saline with tween-20 (PBST). Slides were blocked in 5\% Bovine Serum Albumin in PBS for $30 \mathrm{~min}$ at room temperature. Slides were then stained with the primary antibody (1:100 in PBST) for $60 \mathrm{~min}$ at room temperature followed by three washes in PBST. Protected from light, slides were stained with the secondary antibody (1:100 in PBST) and DAPI for $60 \mathrm{~min}$ at room temperature. Slides were washed three more times in PBST then mounted Vectashield hardset mounting medium (Vector Laboratories, Burlingame, CA, United States), sealed with clear nail polish, then visualized by fluorescence microscopy. For TUNEL staining, cyrosections of mouse brain tissue 
was stained using a TUNEL Assay Kit - HRP-DAB (ab206386) (Abcam) according to manufacturer's protocol. For HSV-1 VP16 and gB stainings, the M.O.M. (Mouse on Mouse) Immunodetection Kit, Fluorescein (FMK-2201) (Vector Laboratories, Burlingame, CA, United States) was used according to manufacturer's protocol.

\section{Immunohistochemical detection of optineurin in human nervous systemtissues.} Human nervous system tissues analyzed by immunohistochemistry for optineurin expression were derived from autopsies performed at the University of Illinois at Chicago. Autopsies were obtained after informed consent and tissue use conformed to Institutional Review Board (IRB)-approved protocols. Sections of paraffin-embedded human brain tissues were prepared and evaluated using standard histopathologic techniques and neuropathologic diagnostic criteria by a board-certified neuropathologist. For immunohistochemical analysis, $5 \mu \mathrm{m}$ sections of paraffin-embedded formalin fixed tissue were deparaffinized with xylene and rehydrated. Antigen unmasking was performed in $1 \mathrm{X}$ citrate buffer, $\mathrm{pH} 6.0$ just below boiling for $10 \mathrm{~min}$. Reaction container and slides were allowed to cool for $20 \mathrm{~min}$. The slides were then removed from reaction container, washed in $\mathrm{dH} 2 \mathrm{O}$, incubated in $3 \%$ hydrogen peroxide for $10 \mathrm{~min}$, washed in $\mathrm{dH} 2 \mathrm{O}$, and washed in PBS for $5 \mathrm{~min}$. Immunohistochemisty was performed using the Vectastain Elite ABC-HRP Kit (Rabbit IgG) (PK6101) using a 1:300 dilution of Optineurin C-TERM rabbit polyclonal antibody (Cayman). Detection was achieved by incubating slides in ImmPACT DAB (SK4105). Counterstaining was performed by incubating slides in a $50 \%$ hematoxylin solution for $30 \mathrm{~s}$. Slides were then dehydrated with ethanol and mounted with Permount.

Flow cytometry. Cells were removed from culture plate using HANK's dissociation buffer. Cells were washed in PBS with 5\% FBS then fixed with 4\% paraformaldehyde. Cell were washed again in PBS 5\% FBS then resuspended in the same buffer for flow cytometry on an Accuri C6 flow cytometer (BD Sciences). Data collection was performed using the BD Accuri C6 Plus v1.0 software. Histograms were prepared in FlowJo v10.0.7 software. For brainstem analysis, tissue was dissociated in $100 \mu \mathrm{L}$ of $5 \mathrm{mg} / \mathrm{mL}$ collagenase IV (Sigma Aldrich) in OptiMem (Gibco). The tissue was spun down at $500 \times g$ for $1 \mathrm{~min}$ and resuspended in FACS buffer (PBS, 1\% BSA). The cell suspension was filtered using $100 \mu \mathrm{m}$ sterile filter, then blocked using TruStain FcX (BioLegend) according to manufacturer's protocol. After 2 rounds of centrifugations and washes in 1 mL FACS buffer, the samples were stained for CD3, CD4, and CD8 using $1 \mu \mathrm{L}$ of antibody and $100 \mu \mathrm{L}$ (10\%) of the cell suspension for $1 \mathrm{~h}$. After 2 rounds of centrifugations and washes in $1 \mathrm{~mL}$ FACS buffer, the samples were resuspended in $1 \mathrm{~mL}$ FACS buffer and immediately analyzed on an Accuri C6 flow cytometer (BD Sciences).

Apoptosis detection by flow cytometry. Optn $+/+$ and Optn-/- HeLa cells were infected with HSV-1 at $0.1 \mathrm{MOI}$ and harvested at $24 \mathrm{hpi}$. FITC Annexin V/Dead Cell Apoptosis Kit (InvitrogenTM) was used to detect apoptosis. As per the manufacturer protocol the sample pellets were washed with cold PBS and then suspended in 1x annexin binding buffer. The suspension was mixed with $5 \mu \mathrm{L}$ FITC Annexin $\mathrm{V}$ and $1 \mu \mathrm{L}$ of a propidium iodide solution $(100 \mu \mathrm{g} / \mathrm{mL})$ for $15 \mathrm{~min}$ in the cold. The mixture was diluted with $1 \mathrm{X}$ annexin binding buffer. Further flow cytometry (BD AccuriTM C6 Plus flow cytometer) was used to analyze the staining for Annexin V and propidium iodide. Flow data was analyzed using FlowJo software (Tree Star Inc.).

Plaque assays. After scraping cells from a culture dish cell pellets were resuspended in $1 \mathrm{~mL}$ of Opti-Mem. Cell suspension were sonicated for a $5 \mathrm{~s}$ pulse at $25 \%$ amplitude then immediately serially diluted in Opti-MEM. For tissue samples, the tissue was place in $1 \mathrm{ml}$ of Opti-MEM and sonicated as described. Dilutions are added to Vero cell monolayers and incubated at $37{ }^{\circ} \mathrm{C}$ and $5 \% \mathrm{CO}_{2}$ for $2 \mathrm{~h}$. Following incubation the virus dilution is aspirated and fresh medium (DMEM, 10\% FBS, $1 \%$ Penn/Strep, $5 \%$ methylcelluose $\mathrm{w} / \mathrm{v}$ ) is added to the culture dish. Cells are returned to incubator $\left(37^{\circ} \mathrm{C}\right.$ and $\left.5 \% \mathrm{CO} 2\right)$ for $\sim 3$ days or until plaques can be observed. Monolayers are fixed by adding methanol directly to the medium for $10 \mathrm{~min}$ at room temperature and then aspirating the methanol-containing medium. The cells are stained with crystal violet (70\% water, $20 \%$ ethanol, $10 \%$ crystal violet stock [ $1 \mathrm{~g} / 100 \mathrm{~mL}$ crystal violet in $20 \%$ ethanol] $)$ for $1 \mathrm{~h}$, then aspirated and dried to visually count plaques under a microscope.

Immunoprecipitation. Cells were scraped from culture dishes and centrifuged to pellet cells. Cell pellets were lysed on ice for $1 \mathrm{~h}$ in immunoprecipitation (IP) buffer ( $250 \mathrm{mM} \mathrm{NaCl}, 50 \mathrm{mM}$ Tris, $0.5 \mathrm{mM}$ EDTA, $0.5 \% \mathrm{NP}-40$ ) with protease/phosphatase inhibitor added. For the anti-VP16 immunoprecipitation, $10 \mathrm{mM} \mathrm{N}$ Ethylmaleimide was added to the IP Buffer immediately before use. Lysates were centrifuged to remove cell debris and the soluble portion was precleared with Isotype control antibody (Santa Cruz) and protein A/G conjugated beads (Santa Cruz) for $1 \mathrm{~h}$ with agitation at $4{ }^{\circ} \mathrm{C}$. Beads were pelleted by centrifugation and lysates were moved to new tubes. The lysates were then incubated with isotype or specific antibody ( $5 \mu \mathrm{g}$ per $1.7 \mathrm{~mL}$ tube with $1 \mathrm{~mL}$ lysate) on ice for $1 \mathrm{~h} .20 \mu \mathrm{L}$ of protein $\mathrm{A} / \mathrm{G}$ beads were added and samples were agitated at $4{ }^{\circ} \mathrm{C}$ overnight. The beads containing the immunoprecipitated proteins were pelleted by centrifugation and the unbound portion decanted. The beads were washed four times in IP buffer prior to processing for immunoblot analysis.

Immunoblot. Cells were scraped from cell culture dish and cells collected in $1.7 \mathrm{~mL}$ tubes were centrifuged. Cell pellets were lysed on ice for $30 \mathrm{~min}$ using RIPA buffer (Sigma) with added protease and phosphatase inhibitor unless otherwise stated for immunoprecipitation. Lysates were centrifuged to pellet debris and the soluble fraction was mixed with NuPage LDS Sample Buffer (Life Technologies) and $\beta$ mercaptoethanol. Samples were incubated on a heat block at $95^{\circ} \mathrm{C}$ for $10 \mathrm{~min}$. Electrophoresis was performed using a NuPage $4-12 \%$ gradient polyarcrylamide gel (Life Technologies) with NuPage MOPS running buffer (Life Technologies) at $70 \mathrm{~V}$ at room temperature. Proteins were transferred to a PVDF membrane using an iBlot 2 system (Thermo Fisher Scientific). Membranes were blocked for $1 \mathrm{~h}$ in blocking buffer (5\% milk in tris-buffered saline with $0.1 \%$ tween-20 (TBST)) followed by overnight primary incubation (1:1000-1:5000 in blocking buffer) with gentle rocking at $4{ }^{\circ} \mathrm{C}$. Membranes were washed three times in TBST before incubation with secondary anti-rabbit or anti-mouse antibody conjugated to HRP $(1: 10,000$ in blocking buffer) for $2 \mathrm{~h}$ at room temperature. ECL Femto Substrate (Thermo Fisher Scientific) was used to develop blots and bands were visualized using a Quant 4000 (General Electric) and densitometry analysis was performed in ImageQuant TL and ImageJ software. GAPDH was used as a reference control for sample loading.

Entry assay. In all, $2 \times 10^{4}$ cells per well were plated in a 96-well format. Cells were confluent after incubation overnight and were then infected at multiple MOIs with gL86 HSV-1. 6 hpi the medium was aspirated, and the cells were washed with PBS, In all, $100 \mu \mathrm{L}$ of $\beta$-galactosidase substrate $(0.5 \% \mathrm{NP} 40,3 \mathrm{mg} / \mathrm{mL}$ O-nitro-phenyl- $\beta$ D-galactopyranoside [Thermo Fisher Scientific]) was added to each well and plated were incubated at $37^{\circ} \mathrm{C}$ for $2 \mathrm{~h}$. Colorimetric reaction signal was measured at $410 \mathrm{~nm}$ using a GENESIS Pro Plate reader. Alternatively, cells were plated in a 12 well format and infected with gL86 HSV-1 at the same MOIs for the same amount of time. X-gal was used to develop the reaction and the plate was scanned to compare the color visually.

Infections. Infections were performed by suspending HSV-1 from a thawed stock in Opti-MEM and adding this mixture to cell cultures. Cells are incubated with the virus for $2 \mathrm{~h}$ before medium is removed and virus-free growth medium is added to cells. In total, 17 strain was used for in vitro experiments unless otherwise specified.

Transfection. HeLa cells were plated to be $60-70 \%$ confluent in six-well format. In all, $2 \mu \mathrm{L}$ Lipofectamine 2000 (Thermo Fisher Scientific) was used to transfected $2 \mu \mathrm{g}$ of plasmid as directed by the manufacturer's protocol in Opti-MEM. In total, $6 \mathrm{~h}$ after transfection cells were washed in growth medium and then incubated for $18 \mathrm{~h}(24 \mathrm{~h}$ total). Cells were transferred to imaging dishes or lysates were collected for experimental use.

Image analysis. Image analysis was performed in ImageJ software. For time-lapse imaging ImageJ was used to set a color threshold for intense green fluorescence. This threshold allowed the selection and measurement of the infected area relative to total area for each frame, and this data was subsequently graphed in GraphPad Prism. The infection velocity was derived from the change in the infected area between two adjacent time-points, and graphed.

Particle counts were collected in ImageJ by setting color thresholds for each particle type, then using the particle analysis feature in ImageJ to count the number of particles present in the frame.

Colocalization was analyzed in ImageJ. Background fluorescence was subtracted from the raw data of each individual channel image using a rolling ball method with a radius of 30 pixels. Following subtraction, colocalization was quantified by finding the Mander's correlation coefficient (MCC) using the Coloc 2 plugin. The region of interest used was hand-drawn around the cell or the nucleus to determine the MCC for the nucleus or extranuclear region.

Localization of OPTN was analyzed in ImageJ by similarly subtracting background and drawing regions of interest in the raw data images. The selected regions were measured for signal area and total area and these measurements were used to determine the signal density for the nucleus or extranuclear region.

Cytokine profiling. Brainstem or draining lymph node samples were collected from mock or 8 dpi mice and profiled using the MILLIPLEX MAP Mouse Cytokine/Chemokine Magnetic Bead Panel - Immunology Multiplex Assay (MCYTOMAG-70K, Millipore Sigma), with the aid of a Millipore Sigma field application scientist. The measurements were analyzed using a K-means clustering algorithm and plotted as heatmaps using the MIT Broad Institute's Morpheus online tool (https://software.broadinstitute.org/morpheus/).

Statistics and reproducibility. All statistical analysis and graph making was carried out in GraphPad Prism software, except for flow cytometry histograms and dot plots produced in FlowJo software. Error bars represent \pm SEM of at least three independent measurements $(n=3)$. Asterisks denote a significant difference, as determined by two-tailed unpaired Student's $t$ test, Mann-Whitney $U$ test, or 
Logrank test: ${ }^{*} p<0.05 ;{ }^{* *} p<0.01 ;{ }^{* * *} p<0.001$; ns not significant, or two-way ANOVA; ${ }^{* * * *} p<0.0001$. All experiments were repeated independently a minimum of three times.

Ethical approval. The authors have complied with all regulations regarding the use of research animals and the study protocol was approved by the University of Illinois at Chicago Animal Care Committee (protocol: 20-065).

Reporting summary. Further information on research design is available in the Nature Research Reporting Summary linked to this article.

\section{Data availability}

All data required to understand the study has been made available in the material presented. Data and materials used in this study can be made available through contact with the corresponding author. Source data are provided with this paper.

Received: 2 August 2020; Accepted: 11 August 2021;

Published online: 13 September 2021

\section{References}

1. Eimer, W. A. et al. Alzheimer's disease-associated $\beta$-amyloid is rapidly seeded by herpesviridae to protect against brain infection. Neuron 100, 1527-1532 (2018).

2. Readhead, B. et al. Multiscale analysis of independent Alzheimer's cohorts finds disruption of molecular, genetic, and clinical networks by human herpesvirus. Neuron 99, 64-82 (2018).

3. Krueger, G. R. F. et al. Comparison of seroprevalences of human herpesvirus- 6 and -7 in healthy blood donors from nine countries. Vox Sang. 75, 193-197 (1998).

4. Liesegang, T. J. Herpes simplex virus epidemiology and ocular importance. Cornea 20, 1-13 (2001).

5. Rezaie, T. et al. Adult-onset primary open-angle glaucoma caused by mutations in optineurin. Science 295, 1077-1079 (2002).

6. Rosato, P. C. \& Leib, D. A. Neuronal interferon signaling is required for protection against herpes simplex virus replication and pathogenesis. PLOS Pathog. 11, e1005028 (2015).

7. van Lint, A. et al. Herpes simplex virus-specific $\mathrm{CD}^{+} \mathrm{T}$ cells can clear established lytic infections from skin and nerves and can partially limit the early spread of virus after cutaneous inoculation. J. Immunol. 172, 392 (2004).

8. He, L., Chen, L. \& Li, L. The TBK1-OPTN axis mediates crosstalk between mitophagy and the innate immune response: a potential therapeutic target for neurodegenerative diseases. Neurosci. Bull. 33, 354-356 (2017).

9. Li, F. et al. Structural insights into the interaction and disease mechanism of neurodegenerative disease-associated optineurin and TBK1 proteins. Nat. Commun. 7, 12708 (2016).

10. Deng, Z. et al. Autophagy receptors and neurodegenerative diseases. Trends Cell Biol. 27, 491-504 (2017).

11. Lazarou, M. et al. The ubiquitin kinase PINK1 recruits autophagy receptors to induce mitophagy. Nature 524, 309-314 (2015).

12. Liu, Z. et al. Ubiquitylation of autophagy receptor Optineurin by HACE1 activates selective autophagy for tumor suppression. Cancer Cell 26, 106-120 (2014).

13. Richter, B. et al. Phosphorylation of OPTN by TBK1 enhances its binding to $\mathrm{Ub}$ chains and promotes selective autophagy of damaged mitochondria. Proc. Natl Acad. Sci. USA 113, 4039-4044 (2016).

14. Puri, M. et al. Listeriolysin O regulates the expression of optineurin, an autophagy adaptor that inhibits the growth of Listeria monocytogenes. Toxins https://doi.org/10.3390/toxins9090273 (2017).

15. Wild, P. et al. Phosphorylation of the autophagy receptor optineurin restricts Salmonella growth. Science 333, 228-233 (2011).

16. Sundaramoorthy, V. et al. Defects in optineurin- and myosin VI-mediated cellular trafficking in amyotrophic lateral sclerosis. Hum. Mol. Genet. 24, 3830-3846 (2015).

17. Maruyama, H. et al. Mutations of optineurin in amyotrophic lateral sclerosis. Nature 465, 223-226 (2010).

18. Osawa, T. et al. Optineurin in neurodegenerative diseases. Neuropathology $\mathbf{3 1}$, 569-574 (2011).

19. Orvedahl, A. et al. Autophagy protects against Sindbis virus infection of the central nervous system. Cell. Host Microbe 7, 115-127 (2010).

20. Yordy, B., Iijima, N., Huttner, A., Leib, D. \& Iwasaki, A. A neuron-specific role for autophagy in antiviral defense against herpes simplex virus. Cell Host Microbe 12, 334-345 (2012).

21. Sudhakar, C., Nagabhushana, A., Jain, N. \& Swarup, G. NF-kappaB mediates tumor necrosis factor alpha-induced expression of optineurin, a negative regulator of NF-kappaB. PLoS ONE 4, e5114 (2009).
22. Bakshi, S., Taylor, J., Strickson, S., Macartney, T. \& Cohen, P. Identification of TBK1 complexes required for the phosphorylation of IRF3 and the production of interferon $\beta$. Biochem J. 474, 1163-1174 (2017).

23. Mankouri, J. et al. Optineurin negatively regulates the induction of IFNbeta in response to RNA virus infection. PLoS Pathog. 6, e1000778 (2010).

24. Ito, Y. et al. RIPK1 mediates axonal degeneration by promoting inflammation and necroptosis in ALS. Science 353, 603 (2016).

25. Orvedahl, A. et al. Autophagy genes in myeloid cells counteract IFN $\gamma$-induced TNF-mediated cell death and fatal TNF-induced shock. Proc. Natl Acad. Sci USA 116, 16497-16506 (2019).

26. Leib, D. A., Alexander, D. E., Cox, D., Yin, J. \& Ferguson, T. A. Interaction of ICP34.5 with Beclin 1 modulates herpes simplex virus type 1 pathogenesis through control of CD4+ T-cell responses. J. Virol. 83, 12164-12171 (2009).

27. Leger, M. et al. Object recognition test in mice. Nat. Protoc. 8, 2531-2537 (2013).

28. Bouet, V., Freret, T., Dutar, P., Billard, J. M. \& Boulouard, M. Continuous enriched environment improves learning and memory in adult NMRI mice through theta burst-related-LTP independent mechanisms but is not efficient in advanced aged animals. Mech Ageing Dev. 132, 240-248 (2011).

29. Barker, G. R. I. \& Warburton, E. C. When is the hippocampus involved in recognition memory? J. Neurosci. 31, 10721-10731 (2011).

30. Cai, W. H., Gu, B. \& Person, S. Role of glycoprotein B of herpes simplex virus type 1 in viral entry and cell fusion. J. Virol. 62, 2596-2604 (1988).

31. Triezenberg, S. J., Kingsbury, R. C. \& McKnight, S. L. Functional dissection of VP16, the trans-activator of herpes simplex virus immediate early gene expression. Genes Dev. 2, 718-729 (1988).

32. Waisner, H. \& Kalamvoki, M. The ICP0 protein of Herpes Simplex Virus 1 (HSV-1) downregulates major autophagy adaptor proteins sequestosome 1 and optineurin during the early stages of HSV-1 infection. J. Virol. 93, e01258-19 (2019)

33. Thompson, R. L., Preston, C. M. \& Sawtell, N. M. De novo synthesis of VP16 coordinates the exit from HSV latency in vivo. PLoS Pathog. 5, el000352 (2009).

34. Sawtell, N. M. \& Thompson, R. L. De novo herpes simplex virus VP16 expression gates a dynamic programmatic transition and sets the latent/lytic balance during acute infection in trigeminal ganglia. PLoS Pathog. 12, e1005877 (2016).

35. Wong, S. W. et al. Global deletion of Optineurin results in altered type I IFN signaling and abnormal bone remodeling in a model of Paget's disease. Cell Death Differ. 71-84 https://doi.org/10.1038/s41418-019-0341-6 (2020).

36. Edwards, T. G. \& Bloom, D. C. Lund Human Mesencephalic (LUHMES) Neuronal Cell Line Supports Herpes Simplex Virus 1 Latency In Vitro. J Virol. 93, e02210-18 (2019)

\section{Acknowledgements}

We owe thanks to all the members of the Tseng, Valyi-Nagy, and Shukla research groups for supporting the work presented in this study. This research was supported by the National Institutes of Health and National Eye Institute grants; K08-EY021520-02, RO1 EY029426, P30 EY001792 and RO1 EY024710 as well as the Butner Pioneer Award, and Duke Health Scholars. This content is solely the responsibility of the authors and does not necessarily represent the official views of the NIH.

\section{Author contributions}

J.A. led the conceptualization, investigation, analysis, and preparation of written and visual materials. T.Y., C.P., R.S., J.H. and A.A. contributed experimental data, analysis, and review of the manuscript. T.V.N. and B.F. contributed data and interpretation of histology. T.V.N. and D.S. contribution supervision and mentorship of research teams. D.S. and H.T. contributed funding and project administration. H.T. developed the mouse model used in the study.

\section{Competing interests}

The authors declare no competing interests.

\section{Additional information}

Supplementary information The online version contains supplementary material available at https://doi.org/10.1038/s41467-021-25642-z.

Correspondence and requests for materials should be addressed to Deepak Shukla.

Peer review information Nature Communications thanks Ravi Mahalingam and the other, anonymous, reviewer(s) for their contribution to the peer review of this work.

Reprints and permission information is available at http://www.nature.com/reprints

Publisher's note Springer Nature remains neutral with regard to jurisdictional claims in published maps and institutional affiliations. 
(c) (i) Open Access This article is licensed under a Creative Commons Attribution 4.0 International License, which permits use, sharing, adaptation, distribution and reproduction in any medium or format, as long as you give appropriate credit to the original author(s) and the source, provide a link to the Creative Commons license, and indicate if changes were made. The images or other third party material in this article are included in the article's Creative Commons license, unless indicated otherwise in a credit line to the material. If material is not included in the article's Creative Commons license and your intended use is not permitted by statutory regulation or exceeds the permitted use, you will need to obtain permission directly from the copyright holder. To view a copy of this license, visit http://creativecommons.org/ licenses/by/4.0/.

(C) The Author(s) 2021 